\title{
Resonant-state expansion for planar photonic crystal structures
}

\author{
Sam Neale (1) and Egor A. Muljarov* \\ School of Physics and Astronomy, Cardiff University, Cardiff CF24 3AA, United Kingdom
}

(Received 19 August 2019; revised manuscript received 18 January 2020; accepted 22 January 2020; published 22 April 2020)

\begin{abstract}
We present a powerful concept in the field of photonic crystals and metamaterials, applying the resonant-state expansion (RSE) to planar photonic crystal structures. The RSE allows us to understand and quantify optical resonances in photonic crystal structures in terms of the analytic resonant states of a homogeneous planar waveguide. The RSE provides an efficient and reliable tool for accurate calculation of a complete set of the resonant states of a photonic crystal slab, which is required for the correct description and a better understanding of its optical spectra. For the proof of principle, numerical verification of the RSE, and demonstration of its unprecedented accuracy and convergence, an infinite planar photonic crystal slab periodic in one dimension is taken as an example. To illustrate the power of this approach, we consider the mode evolution with the amplitude of the periodic modulation, revealing the role of the guided modes in the formation of bound states in the continuum.
\end{abstract}

DOI: 10.1103/PhysRevB.101.155128

\section{INTRODUCTION}

Photonic crystal structures exhibit a number of fundamental optical properties, such as strong confinement and Bragg scattering of light, which can be used, e.g., for light propagation control in grating couplers [1], photonic integrated circuits [2,3], and beam splitters [4]. The band structure of an idealized photonic crystal (PC), infinitely extended in all directions, is already very complicated [5]. Planar PC systems provide an opportunity for the light trapped within an optical waveguide (WG) to couple to the photonic continuum outside the system [6-9], which makes finding the light eigenmodes of a PC slab an even more challenging task.

The complex transmission spectrum of a PC slab can be intuitively understood as a superposition of a large number of resonances of different linewidth and line shape [7], which can be rigorously described by the resonant states (RSs). Being introduced in quantum mechanics nearly a century ago [10,11], the RSs in electromagnetics are the discrete eigenmodes of an optical system - solutions to Maxwell's wave equation with outgoing boundary conditions (BCs) [12]. Physically, RSs describe a culmination of various constructive and destructive interferences of waves due to multiple reflections within the optical system. The RS eigenfrequency is generally complex, with the quality factor ( $Q$ factor) being the half of the ratio of its real to imaginary part. In planar systems, the RSs include, as a special case, modes with purely real frequency, such as WG modes formed as a result of the total internal reflection. Additionally, a typical optical spectrum of a PC slab contains nonresonant features known as Rayleigh-Wood anomalies [13], which are caused by the opening of new diffraction orders into free space. Mathematically, they correspond to the branch cuts in the complex frequency plane caused by the square root in the light dispersion which can be represented as

*Egor.Muljarov@astro.cf.ac.uk a continuum of modes that lie along the cuts $[14,15]$ and that are similar to the RSs. In purely numerical approaches, such as the finite-difference time-domain calculations with perfectly matched layers used in [16], these cuts can be observed in a form of isolated resonances, or cut modes. In some cases, these continua of modes can even be entirely eliminated from the spectral representation $[17,18]$.

The positions and linewidths of resonances can be modified, e.g., by introducing imperfections or changes to the permittivity. This makes the RSs of particular importance for sensing applications, ranging from measuring the refractive index and chirality of a medium [19-21] to biosensing of individual molecules and atoms [22-24]. RSs have also found applications in miniature lasers [25] and low-loss guiding of light in photonic crystal fibers [2], to name a few. The concept of RSs (also known as "quasinormal modes") is widely used in the literature as a natural tool for understanding the optical properties of micro- and nano-resonators (see, e.g., a recent review [26]).

The resonant-state expansion (RSE) is a novel rigorous approach developed in electrodynamics [27] for calculating the RSs of an optical system. Using a complete set of the RSs of a simpler system as a basis, the RSE performs a mapping of Maxwell's wave equation onto a linear eigenvalue problem, which determines the RSs of the complex system of interest. In addition to a higher numerical efficiency compared to other computational methods, such as finite difference in time domain, finite element, and Fourier modal method, as demonstrated in $[15,28]$, the RSE provides an intuitive physical picture of resonant phenomena, capable of explaining features observed in optical spectra. Also, unlike other approaches, the RSE guarantees the completeness of the set of the RSs found within the selected spectral range, provided that the basis set used as input for the RSE is also complete. The latter is easy to achieve and to verify when choosing the basis system to be analytically solvable. Other approaches, in turn, are not able to guarantee that all relevant modes are found and that there are 
no spurious solutions. The effect of other modes, not included in the optical spectrum, is commonly treated as a background contribution adjusted with a number of fit parameters [29,30].

So far, the RSE has been applied to finite open optical systems of different geometry and dimensionality [14,28,31], as well as to homogeneous [18,32] and inhomogeneous planar waveguides [15]. Recently, the RSE was generalized to systems with frequency dispersion of the permittivity [33] and later on to magnetic, chiral, and bianisotropic optical systems [34], enabling its further application to metamaterials. The RSE has also been used in first order of the perturbation theory for PC structures to describe refractive index sensing [20], and a rigorous analytic mode normalization in PC structures has been presented in [20,21]. However, the RSE has never been applied in full to PC systems.

In this paper, we develop a photonic crystal RSE (PC$\mathrm{RSE}$ ), a rigorous approach for the accurate calculation of RSs in planar PC structures. The key idea of the PC-RSE is to use the analytically solvable homogeneous slab as a basis system and to treat a PC structure as a periodic modulation on top of the slab. This idea echoes back to the famous nearly free electron model in the solid-state theory [35]. However, unlike states of a free electron, the basis RSs of an open optical system are generally leaky (having finite $Q$ factors), which makes the implementation of the same idea in optics entirely different.

The idea of using the eigenmodes of a homogeneous slab has been already implemented as a guided mode expansion method [36]. Among different methods available in the literature, this approach has been considered as the most efficient way of calculating the eigenmodes of PC structures. It provides, in particular, a powerful tool for optimizing parameters of photonic crystal cavities [37]. This method, however, has a significant disadvantage: A set of the guided modes it uses as a basis is incomplete. Furthermore, it treats an open optical system as an effective closed one, not allowing the eigenfrequencies to be complex. This breach has been patched by introducing an additional procedure, similar to Fermi's golden rule, for an approximate treatment of light leakage from the system resulting in finite $Q$ factors of modes [36]. Our approach instead adds to this incomplete set of guided modes all the missing RSs having finite $Q$ factors, as well as the cut modes responsible for the Rayleigh-Wood anomalies observed in optical spectra. Having a complete set of modes generated by the RSE allows us to quantify precisely any optical observable [38-40], such as the transmission, reflection, scattering, and extinction of light. It is also expected that the PC-RSE will become a precise and efficient tool for calculating high $Q$-factor modes in PC cavities [41].

The periodicity of a PC structure mixes all possible Bragg harmonics. Therefore, the basis RSs have to be taken with different in-plane wave numbers. As a result, the dyadic Green's function of the set of Maxwell's equations has branch cuts in the complex frequency plane which have to be taken into account in the PC-RSE along with the RSs. This presents the major complication of the PC-RSE which we have dealt with by splitting the cuts into series of discrete, artificial cut modes added for completeness to the basis RSs, as it was done, e.g., in $[14,15]$.

A significant technical advantage of treating periodic modulations of a homogeneous slab as perturbations is that all the diagonal elements of the perturbation matrix are vanishing due to periodicity (without homogeneous perturbation). This guarantees a low level of numerical errors even for small basis sizes and strong periodic modulations, as we show in this paper. Using for illustration, a dielectric slab in a vacuum periodically modulated in one dimension (1D), we demonstrate the accuracy and efficiency of the PC-RSE in finding the RSs of PC structures.

For verification of the PC-RSE, we compare it with the scattering matrix method (SMM) [6,7], also known in the literature as Fourier modal method [42-45], which has been considered in the literature as the most accurate and reliable way of calculating the optical spectra of infinitely extended periodic open systems. In fact, the SMM is asymptotically exact, having the total number of the Bragg diffraction channels taken into account as the only parameter of the method. However, when used for finding the RSs of the system, the SMM is limited by rather low numbers of Bragg channels, struggling to find all modes in a given frequency range and often returning spurious modes. Recently, a so-called "mode expansion method" has been introduced in [46] for an accurate calculation and qualitative study of bound states in the continuum (BICs) in PC slabs. We note, however, that this method, which appeared under a new name, is nothing else than the well-known SMM.

The PC-RSE presented here is not only a simple numerical tool that allows us to accurately calculate a complete set of the RSs of a planar PC slab with a complicated structure. It is a powerful concept in the field of PC systems revealing the origin and the properties of their RSs. To demonstrate this, we study BICs of a dielectric PC slab with 1D grating. Such modes, having infinite $Q$ factors while residing inside the continuum, were predicted in nonrelativistic quantum systems almost a century ago [47] but have only recently become a subject of particular interest [48-50] and have been observed in optics [51]. We show in particular how BICs are formed in PC structures and how different types of basis RSs of a homogeneous slab contribute to BICs and other resonances of the PC slab, such as quasiguided [7] and Fabry-Pérot (FP) modes.

\section{FORMALISM OF THE PC-RSE}

Consider a PC slab occupying the region $|z| \leqslant a$, where $z$ is the coordinate in the growth direction. Assuming the permittivity and the permeability are isotropic everywhere the electric field $\mathbf{E}$, magnetic field $\mathbf{H}$, and the frequency $\omega$ of a given RS of the PC slab satisfy the following Maxwell equations (the speed of light $c=1$ ):

$$
\begin{gathered}
\nabla \times \mathbf{E}=\omega(\mu+\Delta \mu) i \mathbf{H}, \\
\nabla \times i \mathbf{H}=\omega(\varepsilon+\Delta \varepsilon) \mathbf{E},
\end{gathered}
$$

which have to be solved together with outgoing wave BCs outside the PC slab. Here, we have explicitly separated the total permittivity (permeability), periodic in $x$ direction with the period $d$, into a homogeneous part $\varepsilon(z)[\mu(z)]$ and a periodic part $\Delta \varepsilon(x, z)[\Delta \mu(x, z)]$, obeying

$$
\Delta \varepsilon(x+d, z)=\Delta \varepsilon(x, z), \quad \Delta \mu(x+d, z)=\Delta \mu(x, z) .
$$

For the purpose of a clearer illustration of our approach, we consider the case of the transverse electric (TE) and transverse 
magnetic (TM) polarizations not coupled to each other, which is achieved by assuming that the $y$ component of the in-plane momentum is zero. We note, however, that generalization to the case of a nonzero $y$ component of the momentum and to 2D periodicity of the $\mathrm{PC}$ slab is straightforward, and the whole formalism remains essentially the same as presented here.

Since the TE and TM polarizations are not coupled, each polarization can be treated separately. However, due to the symmetry of Maxwell's equations (1) and (2) with respect to a simultaneous exchange of $\mathbf{E} \leftrightarrow i \mathbf{H}, \varepsilon \leftrightarrow \mu$, and $\Delta \varepsilon \leftrightarrow \Delta \mu$, it is sufficient to treat only one of the two polarizations, for example, the TE polarization, while retaining the permeability in all results, even if all the constituent materials are nonmagnetic.

For the TE polarization, Maxwell's equations (1) and (2) reduce to

$$
(\hat{L}+\Delta \hat{L}) \mathbf{F}=0,
$$

where the vector field $\mathbf{F}$ is formed from three nonvanishing components of the electric and magnetic fields

$$
\mathbf{F}=\left(\begin{array}{c}
E_{y} \\
i H_{x} \\
i H_{z}
\end{array}\right)
$$

and

$$
\begin{gathered}
\hat{L}(x, z ; \omega)=\omega \hat{P}(z)-\hat{D}(x, z), \\
\Delta \hat{L}(x, z ; \omega)=\omega \Delta \hat{P}(x, z)
\end{gathered}
$$

are linear operators, consisting of the generalized permittivity [34]

$$
\hat{P}(z)=\left(\begin{array}{ccc}
\varepsilon(z) & 0 & 0 \\
0 & \mu(z) & 0 \\
0 & 0 & \mu(z)
\end{array}\right)
$$

the curl operator

$$
\hat{D}(x, z)=\left(\begin{array}{ccc}
0 & \partial_{z} & -\partial_{x} \\
-\partial_{z} & 0 & 0 \\
\partial_{x} & 0 & 0
\end{array}\right)
$$

and the perturbation

$$
\Delta \hat{P}(x, z)=\left(\begin{array}{ccc}
\Delta \varepsilon(x, z) & 0 & 0 \\
0 & \Delta \mu(x, z) & 0 \\
0 & 0 & \Delta \mu(x, z)
\end{array}\right) .
$$

Owing to the periodicity, the wave function $\mathbf{F}$ obeys Bloch's theorem,

$$
\mathbf{F}(x+d, z)=\mathbf{F}(x, z) e^{i p d},
$$

determining the quasimomentum $p$ in the $x$ direction. We therefore solve the Maxwell equations (4) for the given $p$, using a periodic dyadic Green's function (GF) $\hat{G}_{p}\left(x ; z, z^{\prime}\right)$ of the homogeneous slab:

$$
\mathbf{F}(x, z)=-\frac{\omega}{d} \int_{d} d x^{\prime} \int d z^{\prime} \hat{G}_{p}\left(x-x^{\prime} ; z, z^{\prime}\right) \Delta \hat{P}\left(x^{\prime}, z^{\prime}\right) \mathbf{F}\left(x^{\prime}, z^{\prime}\right),
$$

where $\int_{d} d x$ implies integration over any period interval. This GF has the same value of $p$ and satisfies Maxwell's equations with a periodic array of sources:

$$
\hat{L}(x, z ; \omega) \hat{G}_{p}=\hat{\mathbf{1}} \delta\left(z-z^{\prime}\right) d \sum_{m} e^{i p m d} \delta\left(x-x^{\prime}-m d\right),
$$

where $m$ is an integer and $\hat{\mathbf{1}}$ is the $3 \times 3$ unit matrix. Using Bloch's theorem again, the periodic GF can be written as

$$
\hat{G}_{p}\left(x ; z, z^{\prime}\right)=\sum_{g} \hat{\mathcal{G}}_{p+g}\left(z, z^{\prime}\right) e^{i(p+g) x},
$$

where

$$
g=\frac{2 \pi m}{d}
$$

and $\hat{\mathcal{G}}_{p}\left(z, z^{\prime}\right)$ is another, $x$-independent GF of the homogeneous slab satisfying an equation

$$
\hat{\mathcal{L}}_{p}(z ; \omega) \hat{\mathcal{G}}_{p}\left(z, z^{\prime}\right)=\hat{\mathbf{1}} \delta\left(z-z^{\prime}\right),
$$

with a modified operator

$$
\hat{\mathcal{L}}_{p}(z ; \omega)=\omega \hat{P}(z)-\hat{\mathcal{D}}_{p}(z),
$$

which consists of the homogeneous generalized permittivity $\hat{P}(z)$ given by Eq. (8) and the curl operator $\hat{\mathcal{D}}_{p}(z)$ given by Eq. (9) with $\partial_{x}$ replaced by $i p$ : $\hat{\mathcal{D}}_{p}(z)=\hat{D}_{\partial_{x} \rightarrow i p}$.

The homogeneous GF $\hat{\mathcal{G}}_{p}$ can be written, using the MittagLeffler (ML) theorem [52], in terms of the RSs of the homogeneous slab,

$$
\hat{\mathcal{G}}_{p}\left(z, z^{\prime}\right)=\mathcal{Y}_{n} \frac{\mathcal{F}_{n}(z ; p) \otimes \mathcal{F}_{n}\left(z^{\prime} ;-p\right)}{\omega-\omega_{n}},
$$

where $\otimes$ denotes the dyadic vector product and $\sum$ represents the sum over the RSs of the homogeneous slab, supplemented with an integral over the branch cuts that are caused by the square root in the wave vector when using $\omega$ representation (see Appendix B). $\mathcal{F}_{n}(z ; p)$ is the vectorial wave function of the RS $n$ of the homogeneous slab, satisfying Maxwell's equation

$$
\hat{\mathcal{L}}_{p}\left(z ; \omega_{n}\right) \mathcal{F}_{n}(z ; p)=0
$$

and outgoing BCs, and $\omega_{n}$ is the RS eigenfrequency. Equation (18) is valid if the RSs are normalized according to a general normalization condition [21,34] applied to the homogeneous planar system,

$$
1=\int_{z_{1}}^{z_{2}} \mathcal{F}_{n}^{\dagger} \cdot \hat{P} \mathcal{F}_{n} d z+\left.\left(\mathcal{E}_{n}^{\dagger} \times i \mathcal{H}_{n}^{\prime}-\mathcal{E}_{n}^{\prime} \times i \mathcal{H}_{n}^{\dagger}\right) \cdot \mathbf{e}_{z}\right|_{z_{1}} ^{z_{2}},
$$

where $\mathbf{e}_{z}$ is the unit vector in the $z$ direction, $z_{1}$ and $z_{2}$ are two arbitrary coordinates outside of the system, such that $z_{1} \leqslant-a$ and $z_{2} \geqslant a$, and $\mathcal{F}_{n}^{\dagger}(z ; p)=\mathcal{F}_{n}(z ;-p)$ is the adjoint field. $\mathcal{E}_{n}$ and $\mathcal{H}_{n}$ in Eq. (20) are, respectively, the electric and magnetic fields of the RS $n$, combined together into the vector $\mathcal{F}_{n}=\left\{\mathcal{E}_{n}, i \mathcal{H}_{n}\right\}$ having in general six components which are reduced to only three for TE or TM polarization, in accordance with Eq. (5); $\mathcal{E}_{n}^{\prime}$ and $\mathcal{H}_{n}^{\prime}$ are the frequency derivatives of the analytic continuation of the fields $\mathcal{E}_{n}$ and $\mathcal{H}_{n}$ into the complex frequency plane (for more details, see $[28,34]$ ). Note that in addition to all the RSs lying on the "physical" Riemann sheet of complex frequency, the ML series (18) includes also cut modes, not obeying Eqs. (19) and (20) (for details and derivation, see Appendices A and B). 
Substituting Eq. (14) into Eq. (12) and using the ML expansion (18), we obtain, owing to the completeness of the basis and in agreement with Bloch's theorem, an expansion of the wave function $\mathbf{F}$ of the RS of the PC slab into the RSs of the homogeneous slab

$$
\mathbf{F}(x, z)=\sum_{g} \bigvee_{n} c_{n}^{g} \mathcal{F}_{n}(z ; p+g) e^{i(p+g) x},
$$

where the expansion coefficients are given by

$$
\begin{aligned}
c_{n}^{g}= & -\frac{\omega}{\omega-\omega_{n}^{g}} \frac{1}{d} \int_{d} d x e^{-i(p+g) x} \\
& \times \int d z \mathcal{F}_{n}(z ;-p-g) \cdot \Delta \hat{P}(x, z) \mathbf{F}(x, z) .
\end{aligned}
$$

Then, substituting the expansion (21) into Eq. (22), we arrive at the the key equation of the PC-RSE:

$$
\omega \sum_{g^{\prime}} \sum_{n^{\prime}}\left(\delta_{n n^{\prime}} \delta_{g g^{\prime}}+V_{n n^{\prime}}^{g g^{\prime}}\right) c_{n^{\prime}}^{g^{\prime}}=\omega_{n}^{g} c_{n}^{g},
$$

in which $\delta_{i j}$ is the Kronecker delta, and the matrix elements of the perturbation are defined as

$$
V_{n n^{\prime}}^{g g^{\prime}}=\int \mathcal{F}_{n}(z ;-p-g) \cdot \Delta \hat{P}_{g-g^{\prime}}(z) \mathcal{F}_{n^{\prime}}\left(z ; p+g^{\prime}\right) d z
$$

with $\Delta \hat{P}_{g}(z)$ being the Fourier coefficients of the generalized permittivity perturbation

$$
\Delta \hat{P}_{g}(z)=\frac{1}{d} \int_{d} \Delta \hat{P}(x, z) e^{-i g x} d x
$$

Note that in Eqs. (22) and (23), we have added index $g$ to $\omega_{n}$ in order emphasize the dependence of the basis RS frequencies on the Bragg channel number $g$.

Equation (23) presents a matrix eigenvalue problem, linear in $\omega$ (the eigenfrequency of a perturbed RS of the PC slab) and can be solved simply by diagonalizing a complex symmetric matrix. This equation is very similar to the RSE equation for a finite open optical system [27]. However, the main difference between the two is that Eq. (23) contains a summation over all Bragg channels, labeled by the index $g$. Also, the contribution of the cuts, denoted by the integral, is included in Eq. (23), in the same way as it was done in the RSE applied to 2D open optical systems [14] or to inhomogeneous waveguides [15].

Note that the RSE has been recently formulated for PC systems $[20,21]$, in a form of a perturbation theory treating some modifications to the already existing periodic structure, i.e., using a PC slab as a basis system. It has been applied so far to either weak perturbations, limiting the RSE basis to a single mode [20], or to moderate perturbations of quasidegenerate modes, limiting the basis to such a pair of modes [21]. In this work we consider instead a homogeneous basis containing up to several thousands of modes of the homogeneous basis. This choice of the basis presents a significant advantage in implementation of the RSE. For example, all different Bragg channels are fully isolated in the homogeneous basis, whereas a PC basis has all these channels already coupled together. This has, in particular, a dramatic consequence on the inclusion of branch cuts in the basis, which is impossible to do in practice with Bragg channels mixed up as in the PC basis. Furthermore, owing to the simplicity of the basis system used in the present approach, all of the basis RSs and cut modes are found analytically.

\section{RESULTS}

\section{A. PC-RSE for permittivity perturbations}

We now use the PC-RSE derived in Sec. II to find the RSs of a nonmagnetic PC slab with a periodic modulation of the permittivity. In this case, the perturbation matrix in the TE polarization simplifies to

$$
V_{n n^{\prime}}^{g g^{\prime}}=\int E_{n}^{g}(z) \Delta \varepsilon_{g-g^{\prime}}(z) E_{n^{\prime}}^{g^{\prime}}(z) d z
$$

where

$$
\Delta \varepsilon_{g}(z)=\frac{1}{d} \int_{d} \Delta \varepsilon(x, z) e^{-i g x} d x
$$

and $E_{n}^{g}(z)$ is the electric field (directed along $y$ ) of the homogeneous-slab RS with index $n$ and momentum $p+g$ along $x$. In general, this RS is a solution of the Maxwell wave equation (B7) with the outgoing boundary condition (B15) and the normalization given by Eq. (B16) (see Appendix B). Here, we have added index $g$ to the electric field $E_{n}^{g}$ and the eigenfrequency $\omega_{n}^{g}$, in order to distinguish different Bragg channels contributing to the PC-RSE.

Note that in order to treat a permittivity perturbation in the $\mathrm{TM}$ polarization, one should instead set $\Delta \varepsilon=0$ in Eq. (24) and use $\Delta \mu$ for the modulation of the permittivity, along with replacements $i H_{x} \rightarrow E_{x}$ and $i H_{z} \rightarrow E_{z}$ in the unperturbed wave function $\mathcal{F}_{n}(z ; p)$.

As a basis system, we choose a homogeneous dielectric slab in a vacuum, of thickness $2 a$, permittivity $\epsilon>1$, and permeability $\mu=1$. The full permittivity profile of the slab system is given by Eq. (C1), and the basis RSs and cut densities are provided in Appendix C. In practical use of Eq. (23) we apply a cut discretization, described in detail in Appendix D2, which modifies the PC-RSE equation to

$$
\omega \sum_{\bar{n}^{\prime} g^{\prime}}\left(\delta_{\bar{n} \bar{n}^{\prime}} \delta_{g g^{\prime}}+V_{\bar{n} \bar{n}^{\prime}}^{g g^{\prime}}\right) c_{\bar{n}^{\prime}}^{g^{\prime}}=\omega_{\bar{n}}^{g} c_{\bar{n}}^{g}
$$

with an integer index $\bar{n}$ labeling both the RSs and the cut modes [see Eq. (D8)]. Within the slab, $|z| \leqslant a$, the electric fields of the RSs and cut modes are described by the same functions

$$
E_{\bar{n}}^{g}(z)=B_{\bar{n}}^{g}\left[e^{i q_{\bar{n}}^{g} z}+(-1)^{\bar{n}} e^{-i q_{\bar{n}}^{g} z}\right]
$$

with the normalization constants for the RSs and cut modes given by Eqs. (C9) and (D10), respectively. The mode parity is determined by the parity of $\bar{n}$ via the coefficient $(-1)^{\bar{n}}$ taking the values of +1 or -1 . The eigenfrequencies $\omega_{\bar{n}}^{g}$ are determined by the secular equation (C7) for the RSs and by Eq. (D5) for the cut modes. Furthermore, the link between the mode frequency $\omega_{\bar{n}}^{g}$ and the wave number $k_{\bar{n}}^{g}$ in vacuum and $q_{\bar{n}}^{g}$ in the medium is provided by the following light dispersion relations:

$$
\begin{gathered}
\left(\omega_{\bar{n}}^{g}\right)^{2}=\left(k_{\bar{n}}^{g}\right)^{2}+(p+g)^{2}, \\
\epsilon\left(\omega_{\bar{n}}^{g}\right)^{2}=\left(q_{\bar{n}}^{g}\right)^{2}+(p+g)^{2} .
\end{gathered}
$$

For illustration purposes and also for the ease of comparison with the SMM, the perturbation of the homogeneous slab 


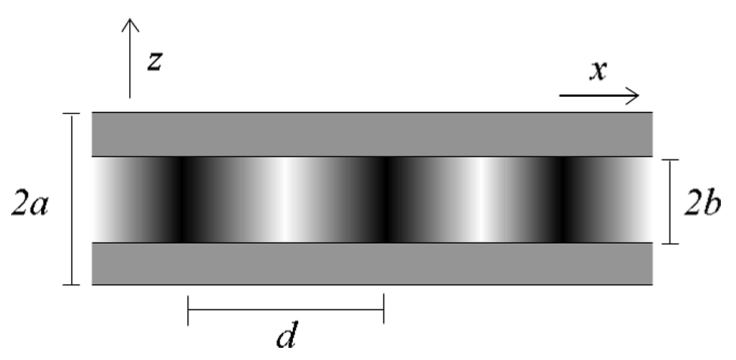

FIG. 1. Schematic of the perturbed system: photonic crystal slab of the total thickness $2 a$, periodically modulated in $x$ direction, with period $d$, within the layer of thickness $2 b$ at the center of the slab.

is taken in the most simple harmonic form

$$
\Delta \varepsilon(x, z)=\left(\alpha+\beta \cos \frac{2 \pi x}{d}\right) \Theta(b-|z|),
$$

where $\Theta(z)$ is the Heaviside step function, $b \leqslant a$, and $\alpha$ and $\beta$ are some parameters (see Fig. 1). We note, however, that the RSE can equally deal with any other shape of the periodic perturbation, not requiring the separation of variables which the perturbation in the form of Eq. (32) possesses. The SMM, in turn, requires this separation. In fact, the transfer matrices that form the scattering matrix are calculated layer by layer through the system (see [7]). Therefore, any system changing smoothly in the growth direction will be approximated by a stack of slices homogeneous in $z$, which are ideally infinitely thin.

For the perturbation given by Eq. (32), the matrix elements take the following explicit form:

$$
V_{\bar{n} \bar{n}^{\prime}}^{g g^{\prime}}=B_{\bar{n}}^{g} B_{\bar{n}^{\prime}}^{g^{\prime}}\left(2 \alpha \delta_{g g^{\prime}}+\beta X_{g-g^{\prime}}\right) b Z_{\bar{n} \bar{n}^{\prime}}^{g g^{\prime}},
$$

where

$$
X_{g}=\delta_{g, g_{1}}+\delta_{g, g_{-1}}
$$

with $g_{ \pm 1}= \pm 2 \pi / d$ and

$$
\begin{aligned}
Z_{\bar{n} \bar{n}^{\prime}}^{g g^{\prime}}= & \left(1+(-1)^{\bar{n}+\bar{n}^{\prime}}\right) \operatorname{sinc}\left[\left(q_{\bar{n}}^{g}+q_{\bar{n}^{\prime}}^{g^{\prime}}\right) b\right] \\
& +\left((-1)^{\bar{n}}+(-1)^{\bar{n}^{\prime}}\right) \operatorname{sinc}\left[\left(q_{\bar{n}}^{g}-q_{\bar{n}^{\prime}}^{g^{\prime}}\right) b\right]
\end{aligned}
$$

with $\operatorname{sinc} z=\sin z / z$.

For homogeneous perturbations, used in Appendix D for the RSE verification and comparison of the $k$ and $\omega$ representations, we use $\alpha \neq 0$ and $\beta=0$. For all periodic perturbations we instead take $\alpha=0$ and $\beta \neq 0$, so that all the matrix elements, Eq. (26), within the same channel $\left(g=g^{\prime}\right)$ are vanishing since $\Delta \varepsilon_{0}(z)=0$, according to Eq. (27). This property yields a quick convergence of the PC-RSE. In fact, since the diagonal elements of the perturbation matrix are all zeros, the first-order contribution of the PC-RSE is vanishing [28]. Then, the lowest-order nonvanishing contribution of the perturbation can only be quadratic in $V_{n n^{\prime}}^{g g^{\prime}}$, making its overall effect quantitatively small and allows the PC-RSE to converge quickly to the exact solution.

\section{B. Basis for the PC-RSE}

The full basis for the PC-RSE consists of an infinite number of RSs and cut modes, taken for all Bragg channels $g=$
$2 \pi m / d$, where $m=0, \pm 1, \pm 2 \ldots$ Periodic perturbations, such as the one given by Eq. (32), introduce coupling between the basis states belonging to different Bragg channels, so that in order to obtain the exact result one needs to take all of them into account simultaneously. In practice, we introduce a truncation, limiting the number of RSs and cut modes for each Bragg channel as well the number of Bragg channels themselves. We do both truncations by introducing a single real parameter $\omega_{\max }$ which defines a circle $|\omega|<\omega_{\max }$ in the complex frequency plane containing the basis RSs and cut modes included in the PC-RSE. For a fixed ratio of the number of RSs and cut modes in the basis, the total number of modes scales as $\omega_{\max }^{2}$ due to the linearly increasing number of channels and the linearly increasing number of modes per channel as $\omega_{\max }$ increases which combine to create a quadratic dependence on $\omega_{\max }$. The set of included RSs, defined in this way, typically contains a large number of WG modes. In fact, only the $m=0$ channel consists of equidistant FP modes which we call in the following leaky modes. All other channels contain WG modes having real frequencies $\omega_{n}^{g}$ within the intervals $(p+g) / \sqrt{\epsilon}<\left|\omega_{n}^{g}\right|<(p+g)$. The number of WG modes within each channel grows linearly with $g$, with the total number increasing quadratically with $g$. The WG modes are separated from FP modes by a series of cut poles of the GF which are positioned vertically down below the branch points at $\omega= \pm(p+g)$. For $\operatorname{Re} \omega>p+g$ and $\operatorname{Re} \omega<-(p+g)$, there are two infinite series of FP modes for each Bragg channel. All this means, in particular, that for a given radius $\omega_{\max }$, the basis includes $2 M+1 \approx \omega_{\max } \sqrt{\epsilon} d / \pi$ channels, most of which consist of only WG modes.

For the purpose of verification of the PC-RSE by comparing it with the SMM, which is presented in Sec. III C below, we use however a different criterion: We limit the number of Bragg channels to $|m| \leqslant M$, where $M$ is a fixed number, and truncate the RSs and cut modes independent of $M$, i.e., using the same number of modes for each selected channel. This is done in order to avoid a computationally expensive root searching within the SMM related to the increase of the $S$-matrix size with $M$. Clearly, for adequate comparison, it is essential to keep the truncation number $M$ the same for both PC-RSE and SMM. However, the necessity to keep $M$ low demonstrates the major weakness of the SMM.

The PC-RSE basis used for the comparison with the SMM is illustrated in Fig. 2, for $p=0$ and $M=5$, showing the eigenfrequencies of both the RSs and cut modes for all selected 11 Bragg channels. Clearly, for $p=0$, the positiveand negative- $m$ channels are degenerate (giving the same RS eigenfrequencies), and both degenerate channels must be included in the basis. Additionally, there are now $2 M$ cuts with discretized cut modes added to the basis. These cut modes are also degenerate for the same reason as the RSs.

\section{Verification of the PC-RSE}

Before applying the PC-RSE, we first consider a homogeneous dielectric slab in vacuum. Taking $\epsilon=6$ and $p a=5$ as an example, we demonstrate numerically in Appendix $\mathrm{C}$ a quick convergence with the basis size $N$ of the ML series for the GF to its exact values, given by the analytic formula (C3). We show in particular that both $k$ and $\omega$ representations of the 


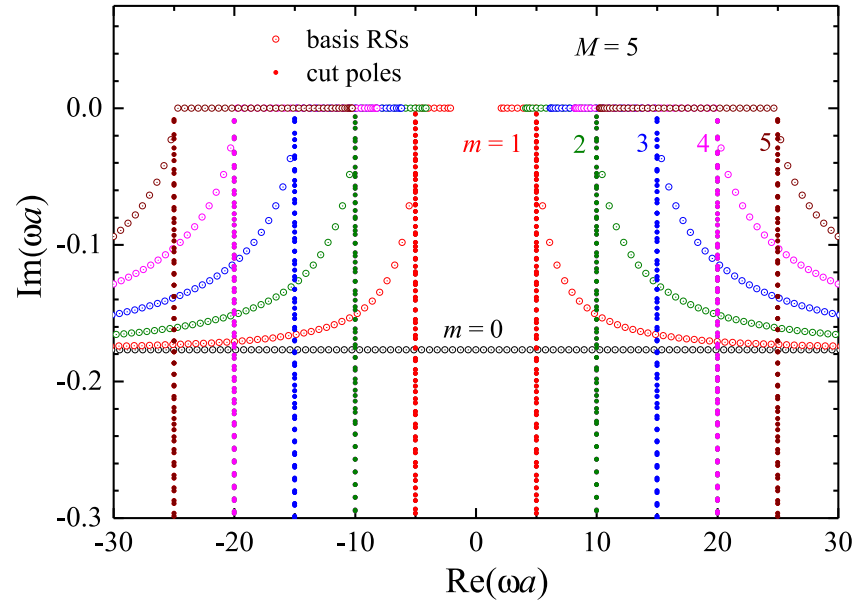

FIG. 2. Basis RSs and cut poles (used for comparison of the PC-RSE with SMM) for $\epsilon=6, \alpha=\beta=0$, and $M=5$ leading to 11 Bragg channels in the basis, each channel labeled with index $m$. The frequencies of the RS and cut modes of channels $m$ and $-m$ are identical.

GF (the first without and the second with cuts in the basis) converge in the same way, with the absolute error scaling as $1 / N^{2}$. The contribution of the cuts to the ML expansion in the $\omega$ representation is taken into account in this case in a form of a numerical integration.

We then apply in Appendix D the RSE to a uniform perturbation of the homogeneous slab for $p \neq 0$, demonstrating for both $k$ and $\omega$ representations a quick convergence of the RSE to the analytic solution available for the core-shell geometry used, with the relative error for the wave numbers scaling as $1 / N^{3}$. The RSE in the $k$ representation essentially reproduces the results of [18]. In the $\omega$ representation applied to homogeneous systems, the cut contribution is taken into account in the RSE in the form of a subset of artificial cut modes obtained by a numerical discretization of the cuts and added to the basis. The procedure of the cut discretization is described in detail in Appendix D 2. These cut modes are then used in the PC-RSE.

Now, in order to verify the PC-RSE, we consider the periodic perturbation (32) with $\alpha=0, \beta=1, d=2 a \pi / 5$, and $b=a / 2$. The unperturbed system is a homogeneous slab in a vacuum of thickness $2 a$ and permittivity $\epsilon=6$. Its RSs and cut modes for TE polarization and $p=0$ are shown in Fig. 2. Perturbed RSs of the PC slab, calculated via the PC-RSE (with and without cut modes) and by the SMM, are shown in Fig. 3 along with all the unperturbed RSs and cut modes present in the displayed spectral range. As already mentioned in Sec. III B, the same truncation of the Bragg channels with $M=5$ was used for both the PC-RSE and SMM.

While the periodic perturbation is not small $(\beta=1)$, leading to a considerable modification of the RSs, one can see in Fig. 3 a very good visual agreement between the SMM and the PC-RSE, even when no cut modes are included in the basis (green crosses $x$ ). In fact, in this case, there is only a slight discrepancy between the two calculations seen for some RSs close to the cuts [see, e.g., region 3 in Fig. 3(b)]. These discrepancies are fully removed when cut modes are included
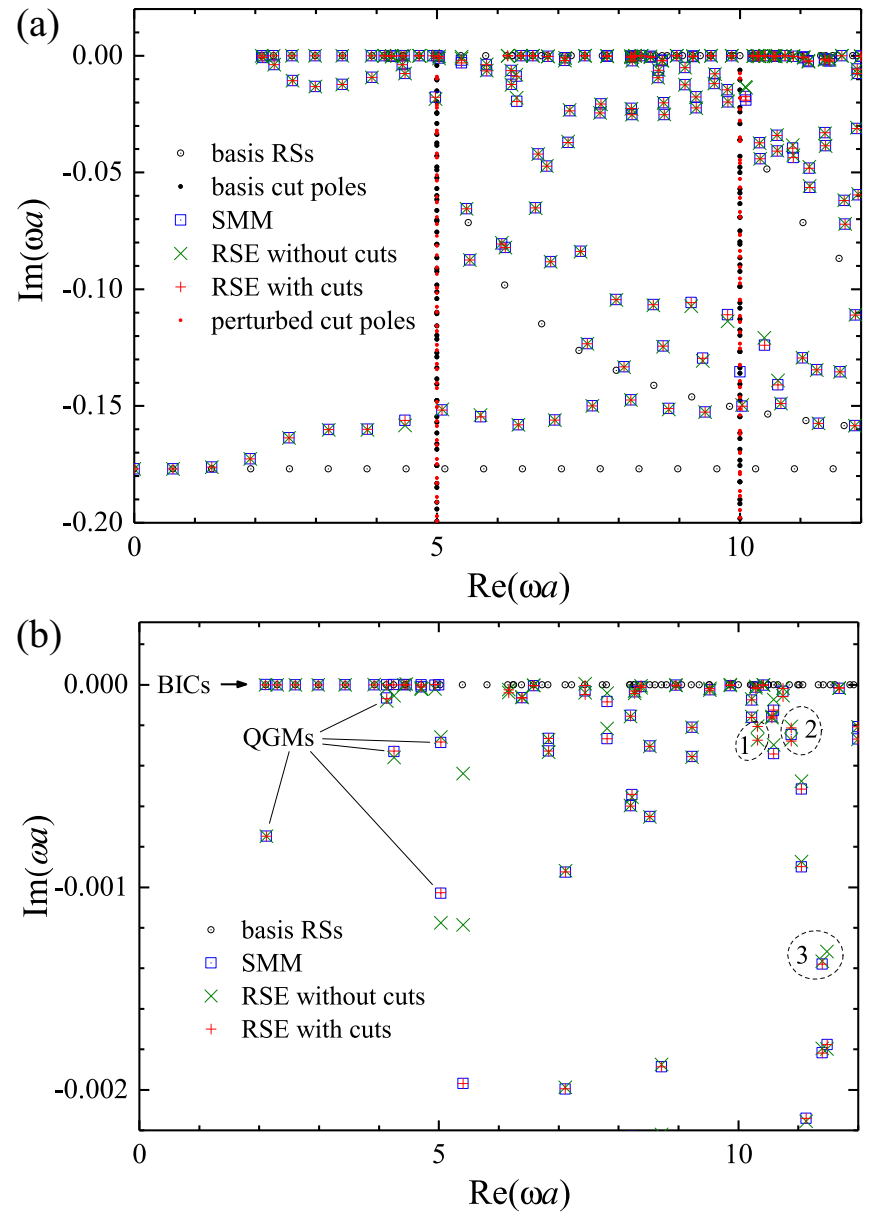

FIG. 3. (a) RS frequencies of a PC slab with $\epsilon=6, \alpha=0$, $\beta=1, b=a / 2$, and $d=2 \pi a / 5$, calculated for $M=5$ using the PC-RSE $(N=1998, F=1)$ with and without cut modes (red and green crosses) and the SMM (blue squares with dots). Unperturbed RSs and both unperturbed and perturbed cut modes are also shown (black circles with dots, black and blue dots, respectively). (b) Zoom of (a) showing RSs near and on the real axis.

in the PC-RSE (red crosses +). Interestingly, the PC-RSE also returns cut modes of the perturbed system, positioned along the same cut lines at $\operatorname{Re}(\omega a)=5$ and 10 , but shifted vertically with respect to their unperturbed positions [compare red and black points in Fig. 3(a)].

To quantify the agreement between the PC-RSE and SMM, we study the relative error for the RS frequencies, which is shown in Fig. 4(a). Increasing the basis size $N$, the error does not improve for some RSs near the cuts, if the cut modes are not included in the basis. Including the cuts, the relative error gradually decreases for all RSs, as the basis size grows. Interestingly, the cut modes do not contribute to all the RSs evenly, and some RSs close to the cuts show rather small errors, which do not improve much when the cut modes are included.

We also show in Fig. 4(b) the relative error of the PC-RSE with respect to itself for a larger basis size, using the single truncation parameter $\omega_{\max }$, as described in Sec. III B. We take in particular the eigenfrequencies calculated for the total basis size of $N_{\text {tot }} \approx 12000$ as the "exact" values in finding the errors 

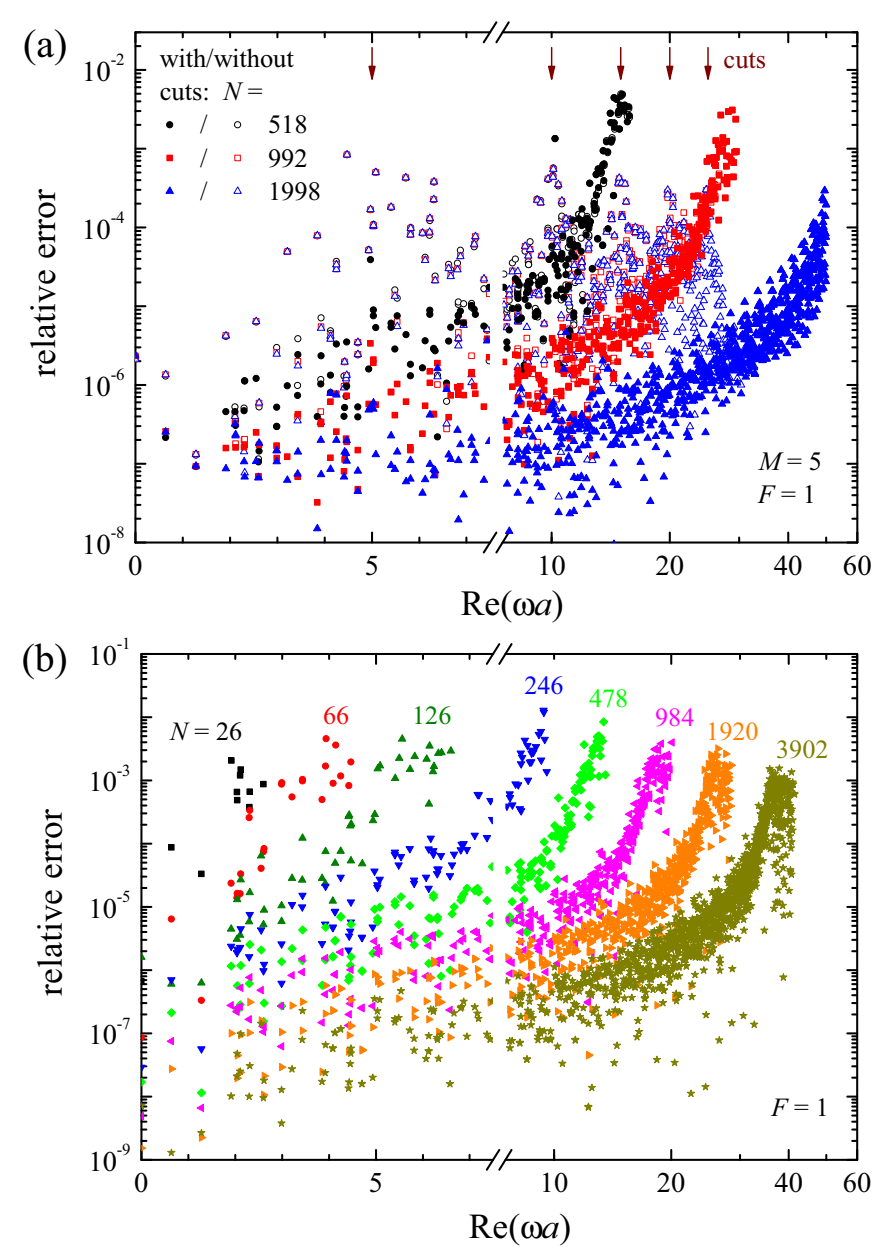

FIG. 4. (a) Relative error of the PC-RSE compared to the SMM result, taking the latter as "exact," calculated with and without cut modes, for different basis sized as labeled, $F=1$, and parameters of the PC slab as in Fig. 3. The basis consists of the closest to the origin modes, with the same number of modes for each Bragg channel, for $M=5$. (b) Relative error of the PC-RSE using the $N \approx 6000$ result as exact, for the same PC slab and the basis size as labeled. The basis consists of all RSs and cut modes within the circle $|\omega|<\omega_{\max }$ in the complex frequency plane.

shown in Fig. 4(b) for different basis sizes. Here, $N_{\text {tot }}=N+$ $F N$, where $N$ and $F N$ are, respectively, the number of RSs and cut modes in the basis. We study the dependence on $F$ of the relative error in Appendix D 2 [see Fig. 13(c)] and show that $F \approx 1$ presents an optimal value for determining the RSs within a rather wide spectral range. This value of $F$ is thus used in the calculations presented in this section.

Looking at the dependence of the error on the basis size, presented in Fig. 4(b), we see that the error decreases by roughly an order as the basis size doubles, which is close to the $1 / N^{3}$ dependence observed for effective 1D systems treated by the RSE [27,28,31]. This demonstrates a high efficiency of the PC-RSE. In fact, its slowest element is matrix diagonalization for which the compute time scales as $N^{3}$. More important is, however, the overall level of errors: even for 26 RSs and no cut modes in the basis, the perturbed RSs of the PC slab are calculated with the accuracy of about or less than $10^{-3}$. The same level of errors is seen in Fig. 4(a) when cut modes are not taken into account, for a large number of RSs found within a much wider spectral range. This can be understood by the already mentioned fact that all diagonal elements $V_{\bar{n} \bar{n}}^{g g}=0$, leading to the effect of the perturbation vanishing in first order, and therefore to a rather low level of corrections and errors.

A large number of the RSs shown in Fig. 3(a) seem to have the imaginary part of the eigenfrequency close to zero. To see this more clearly, we zoom in the view of the imaginary part in Fig. 3(b) by a factor of 100. This allows us to distinguish two types of modes. The first type is known as bound states in the continuum (BICs). These modes, much like WG modes in a planar waveguide, have strictly zero imaginary part and therefore infinite $Q$ factor or lifetime. However, unlike the WG modes, BICs lie in the spectral range where they could (but in reality do not) communicate with the photonic continuum outside of the system. The second type we call quasiguided modes (QGMs) [7] which usually have a very small but nonzero imaginary part of the eigenfrequency (high $Q$ factor), as compared to, e.g., FP modes. This is again due to the dominant role of the WG modes in their formation, like for the BIC, while the small imaginary part is caused by the coupling between the WG and leaky modes (see Sec. III D below for a more detailed analysis of both types of RSs).

We also see in Fig. 3(b) some failures of the SMM, showcasing the superiority of the RSE method. The SMM ultimately relies on the Newton-Raphson method to find the poles of the $S$-matrix $[7,53]$. This means that it uses a small but finite tolerance playing the role of the parameter. If the mode splitting is below the tolerance level, the SMM is unable to resolve them, such as in region 2 of Fig. 3(b). Reducing the tolerance can fix this issue, however, with a potential to generate at the same time spurious solutions at another place or to miss the modes, such as in region 1 of Fig. 3(b). The RSE in turn does not require a tolerance and returns the correct number of RSs in a selected region, neither missing any modes nor producing any spurious solutions. This is an important and unique property of the RSE, following from the completeness of the basis used. Owing to its linearity in $\omega$, the RSE always returns as an output a set of perturbed modes which is also complete. Furthermore, the number of perturbed modes is always equal to the number of basis modes used.

\section{Origin and evolution of the RSs in a PC slab}

Here, we use the high efficiency and accuracy of the PCRSE to find the complete set of the RSs of a PC structure and to study their origin, formation, and further evolution with change of the system parameters. Here we change the most important parameter: the amplitude of the periodic modulation $\beta$. Appendix $\mathrm{F}$ also presents results for varying the thickness of the modulation layer $2 b$ and its period $d$.

Figure 5 shows the evolution of the RSs eigenfrequencies with the increase of the amplitude $\beta$ of the periodic modulation, which we call the perturbation strength. In the $p=0$ case illustrated here, all unperturbed RSs (and cut modes) except the $m=0$ channel are doubly degenerate, as discussed in Sec. III B. The periodic modulation lifts this degeneracy, which is clearly seen in the figure. Furthermore, leaky and FP modes shift upward, increasing their $Q$ factors, while the 

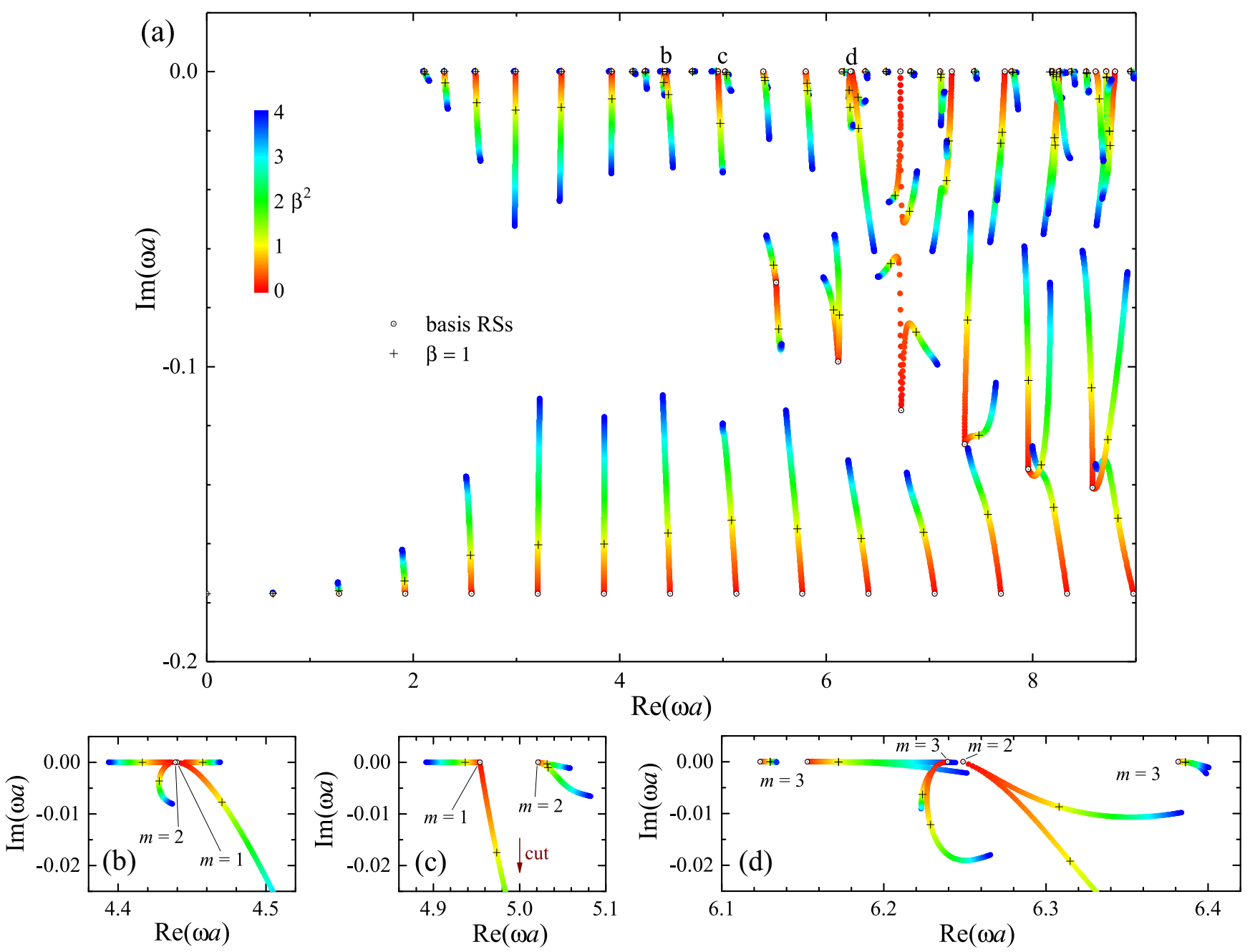

FIG. 5. Evolution of the RS eigenfrequencies of a PC slab for the amplitude of the periodic modulation changing from $\beta=0$ to $\beta=2$ as given by the color code, and the other parameters taken the same as for Fig. 3. Black dotted circles represent the unperturbed modes $(\beta=0)$ and the black crosses the modes shown in Fig. $3(\beta=1)$. (b)-(d) Zoom-in of particular regions in the complex frequency plane close to the real axis, with the values of $m$ indicating the Bragg order of the unperturbed modes.

majority of the RSs originating from WG modes are moving down, away from the real axis, in this way having their $Q$ factors reduced with $\beta$. Overall, this picture demonstrates a complicated mixing of basis modes with both infinite and finite $Q$ factors.

Of particular interest are RSs found close to the real axis of $\omega$. As mentioned in Sec. IIIC above, perturbing the WG modes results in two types of RSs, BICs and QGMs. A closer look provided in Figs. 5(b)-5(d) shows, however, that BICs are formed only within the region $-2 \pi / d<\operatorname{Re} \omega<2 \pi / d$, bound by the cuts of the $m= \pm 1$ Bragg channels. Figure 5(b) demonstrates that each pair of degenerate WG modes within this region produces one BIC and one QGM, the latter losing its quality very quickly with the perturbation strength. Outside this region, we see instead that the RSs originating from the WG modes of the homogeneous slab have either $Q$ factors also quickly decreasing with $\beta$ or very high $Q$ factors weakly depending on the strength of periodic modulation. The latter can be called quasi-BICs, the term which has recently become widely used in the literature for such modes [54,55]. It is also interesting to see a formation of some of the RSs as a result of a rather strong coupling between WG modes which are close in frequency but belong to different Bragg channels (see, e.g., in Fig. 5(b) [Fig. 5(d)] the mode repulsion due to the coupling between WG modes of $m=2$ and 1 [ $m=3$ and 2] channels).

As already discussed in Secs. III A and III C, the perturbation does not contribute in first order (for $\alpha=0$ ), and thus the RSs eigenfrequencies change $\alpha \beta^{2}$ for small $\beta$, in accordance with Eq. (38) of [28], see Fig. 6. However, in the case of the above-mentioned strong coupling between the channels, this linear in $\beta^{2}$ regime takes place only at very low values of $\beta$. Another interesting feature clearly seen in Fig. 6 is that the degenerate pair of basis WG modes producing a BIC-QGM pair shows a linear in $\beta^{2}$ splitting, while any other pair of states, not containing BICs, remains degenerate in this order. This makes BICs even more peculiar.

To understand this and some other properties of BICs, we look at the basis mode contribution to the perturbation of different RSs. We show in Fig. 7 the mode contribution to the BIC-QGM pair selected close to the cut of the first Bragg channel. Other types of modes (FP, leaky, and cut modes) are considered in Appendix E. The size of each circle 

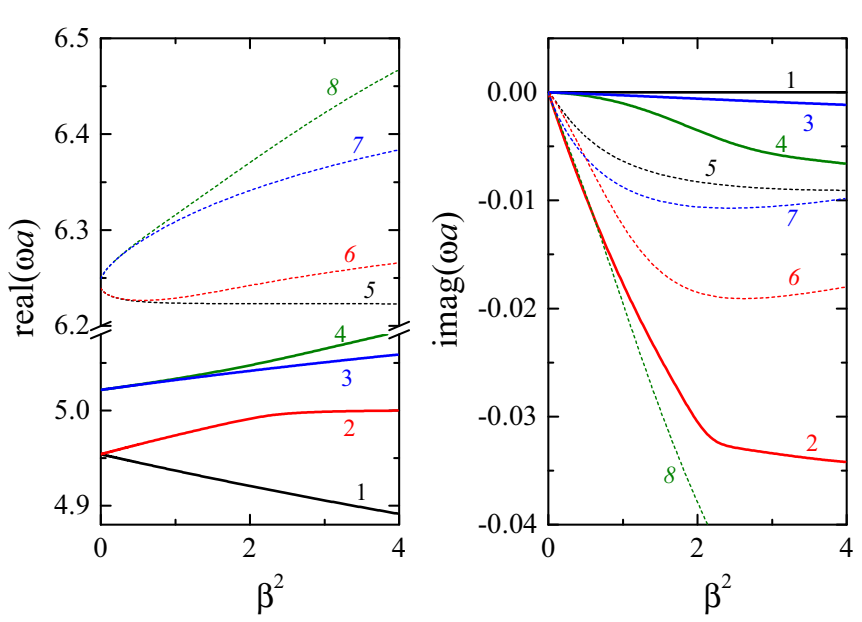

FIG. 6. The real and imaginary parts of the modes shown in Fig. 5(c) and four central modes in Fig. 5(d) as functions of the square of the permittivity modulation amplitude.

represents how much that mode contributes, with the circle area proportional to $\sqrt{\left|c_{n}^{g}\right|}$, chosen (instead of the natural $\left|c_{n}^{g}\right|^{2}$ ) in order to demonstrate more clearly the role of different basis modes. We see that all the basis RSs and cut modes of the same parity as the chosen modes contribute, even though the relevant WG mode has the dominant and indeed very large contribution. It becomes also clear that the main difference between the BIC and the QGM in the studied pair is that the leaky modes of the $m=0$ Bragg channel does not contribute to the BIC. This confirms that the BICs found in this system are symmetry protected [56-59].

In fact, owing to the symmetry of the system, the BIC shown in Fig. 7(a) has a wave function which is odd in the $x$ direction and thus does not couple to the $m=0$ channel containing only even states. In other words, the $m=0$ channel is not present in the subgroup of odd states, and therefore this BIC is not even falling into the continuum: for this subgroup, the continuum starts at the cut positions of the $m= \pm 1$ channels. Furthermore, all doubly degenerate basis states contribute to the QGM (BIC) with the same (opposite) amplitude $c_{n}^{g}= \pm c_{n}^{-g}$, also reflecting selection by symmetry.

Note that the other mirror symmetry of the system, which is in the $z$ direction, also results in the formation of the basis and perturbed RSs of even and odd parity. It is clear, for example, that every other FP mode does not contribute to the states in Fig. 7. Indeed, modes of the opposite parity in $z$ do not couple to each other, and both the QGM and BIC shown in Fig. 7 are of even parity. This implies in particular that the basis size can be halved for this kind of perturbation.

It is also clear from Fig. 7 that the cut modes contribute very little to the perturbed BIC and QGM but are nonetheless required for accuracy. We have seen, in particular, that including even one cut mode, representing the full cut, is far better than not including any cut modes, despite the cut being very badly approximated.

Finally, the observed splitting $\alpha \beta^{2}$ within the BIC-QGM pair (modes 1 and 2 in Fig. 6) can be understood as a result of the leaky modes of the $m=0$ Bragg channel affecting (not affecting) the QGM (BIC). If this channel contributes, the effect of the leaky modes appears already in the second order
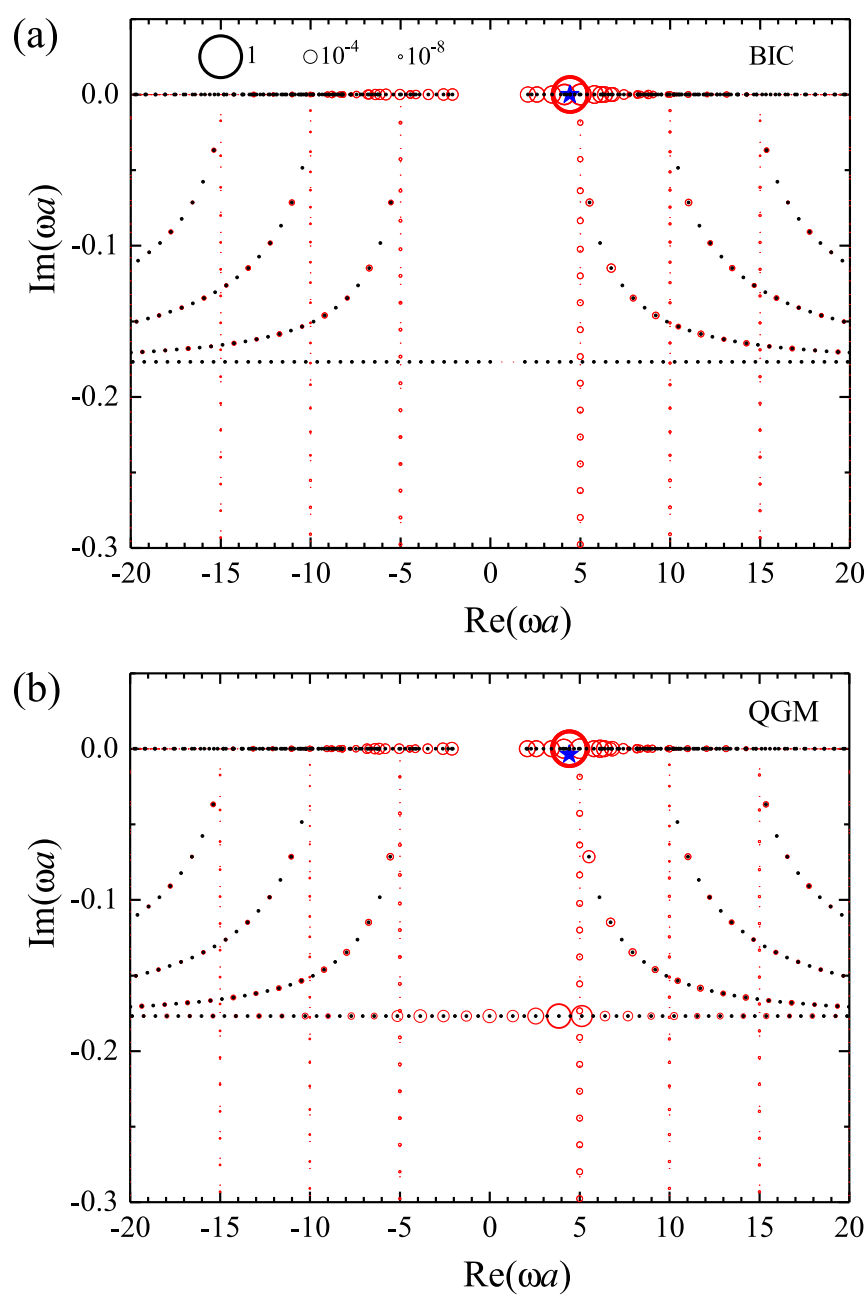

FIG. 7. Basis mode contribution to (a) bound state in the continuum and (b) quasiguided mode, a pair of modes originating from $m=1 \mathrm{WG}$ mode and shown by black crosses in Fig. 5(c). The area of each circle is proportional to $\sqrt{\left|c_{n}^{g}\right|}$. A key showing the relationship between the circle area and $\left|c_{n}^{g}\right|^{2}$ is given as black circles.

in the perturbation $\beta$, and thus causes the splitting of the BIC and QGM in this order.

The studied systems can also support non-symmetryprotected BICs which will be a subject of future studies. These kinds of BICs can form in these systems by tuning their spatial and material parameters, such as the period and strength of the periodic modulation, and the thickness of the substrate layer (see, e.g., [60]).

\section{CONCLUSIONS}

This paper offers a unique and powerful concept in the theory of photonic crystal structures, revealing the nature of resonances in the optical spectra and quantifying them precisely. This concept is based on using the analytical resonant states of a homogeneous slab, along with its cut modes responsible for Rayleigh-Wood anomalies, as the most natural and simple basis for expanding the resonant states of a photonic crystal slab. We present the photonic crystal resonant-state expansion (PC-RSE), capable of doing this accurately and efficiently, 
and illustrate it on examples of TE-polarized modes in a photonic crystal slab with a 1D harmonic modulation. These examples provide a proof of concept and also a verification of the PC-RSE by comparing it with the accurate scatteringmatrix method.

We have presented a general formalism of the PC-RSE and its application to dielectric photonic crystal structures. We have demonstrated that the PC-RSE is an asymptotically exact approach, which (i) depends on a single parameter (truncation frequency $\omega_{\max }$ ) determining the basis size; (ii) guarantees completeness, i.e., has no missing or spurious modes, so that any observable can be represented as a superposition of the modes found; (iii) technically reduces solving Maxwell's wave equation to a matrix diagonalization, thus making the application of the PC-RSE a fully automated and straightforward procedure, not requiring any supervision. The PC-RSE provides an accurate and efficient tool for calculating all physically relevant eigenstates of the PC system within the selected spectral range. In particular, it can be effectively used repeatedly many times or in parallel, in order to investigate a large space of the physical parameters characterizing the system, for revealing and optimizing its fundamental properties. One of the immediate important applications of the PC-RSE is the optimization of photonic crystal cavities, with a correct account of the radiative losses of high-quality modes.

We have also demonstrated how the PC-RSE can be used to study the origin and physical properties of the optical modes. For illustration, we have traced the evolution of the optical modes in a photonic crystal slab as the amplitude of the periodic modulation of the permittivity is increased, starting from a homogeneous slab with no modulation. This allowed us, in particular, to reveal the dominant role of the waveguide modes in the formation of bound states in the continuum and quasiguided modes. Furthermore, the PC-RSE allows us to quantify precisely the contribution of each basis state to the optical mode of interest, which we have also demonstrated in this work.

\section{ACKNOWLEDGMENT}

S.N. acknowledges support by the EPSRC under the DTA scheme.

\section{APPENDIX A: DYADIC GREEN'S FUNCTION OF A HOMOGENEOUS SLAB}

Let us consider arbitrary dependencies of the permittivity $\varepsilon(z)$ and permeability $\mu(z)$ within a homogeneous slab occupying the region $|z| \leqslant a$ and surrounded by a vacuum, so that outside the slab $\varepsilon=\mu=1$. Denoting the components of the dyadic GF as $\left(\hat{\mathcal{G}}_{p}\right)_{i j}=G_{i j}\left(z, z^{\prime}\right)$, Eq. (16) becomes

$$
\left(\begin{array}{ccc}
\omega \varepsilon & -\partial_{z} & i p \\
\partial_{z} & \omega \mu & 0 \\
-i p & 0 & \omega \mu
\end{array}\right)\left(\begin{array}{lll}
G_{11} & G_{12} & G_{13} \\
G_{21} & G_{22} & G_{23} \\
G_{31} & G_{32} & G_{33}
\end{array}\right)=\hat{\mathbf{1}} \delta\left(z-z^{\prime}\right) .
$$

Owing to the reciprocity of the optical system, the GF has the following property:

$$
G_{i j}\left(z, z^{\prime}\right)=G_{j i}^{\dagger}\left(z^{\prime}, z\right)
$$

where the adjoint $\dagger$ means replacing $p \rightarrow-p$.
From Eq. (A1) we obtain for the first column of $\hat{\mathcal{G}}_{p}$ :

$$
\begin{gathered}
\hat{U}(\mu ; \omega) G_{11}\left(z, z^{\prime} ; \omega\right)=\omega \mu(z) \delta\left(z-z^{\prime}\right), \\
G_{21}\left(z, z^{\prime}\right)=-\frac{1}{\omega \mu(z)} \partial_{z} G_{11}\left(z, z^{\prime}\right), \\
G_{31}\left(z, z^{\prime}\right)=\frac{i p}{\omega \mu(z)} G_{11}\left(z, z^{\prime}\right),
\end{gathered}
$$

where the operator $\hat{U}$ is defined as

$$
\hat{U}(\zeta ; \omega)=\zeta(z) \partial_{z} \frac{1}{\zeta(z)} \partial_{z}+\omega^{2} \varepsilon(z) \mu(z)-p^{2}
$$

with $\zeta(z)$ being a weight function. For the second column of $\hat{\mathcal{G}}_{p}$, it follows from Eq. (A1) that

$$
\begin{gathered}
G_{12}\left(z, z^{\prime}\right)=\frac{1}{\omega \chi(z)} \partial_{z} G_{22}\left(z, z^{\prime}\right), \\
\hat{U}(\chi ; \omega) G_{22}\left(z, z^{\prime}\right)=\omega \chi(z) \delta\left(z-z^{\prime}\right), \\
G_{32}\left(z, z^{\prime}\right)=\frac{i p}{\omega \mu(z)} G_{12}\left(z, z^{\prime}\right),
\end{gathered}
$$

where

$$
\chi(z)=\varepsilon(z)-\frac{p^{2}}{\omega^{2} \mu(z)} .
$$

Note that Eq. (A8) is essentially the same as Eq. (A3), provided that $\mu(z)$ is replaced with $\chi(z)$. Finally, for the third column of $\hat{\mathcal{G}}_{p}$ we obtain

$$
\begin{gathered}
\hat{U}(\mu ; \omega) G_{13}\left(z, z^{\prime}\right)=-i p \delta\left(z-z^{\prime}\right), \\
G_{23}\left(z, z^{\prime}\right)=-\frac{1}{\omega \mu(z)} \partial_{z} G_{13}\left(z, z^{\prime}\right), \\
G_{33}\left(z, z^{\prime}\right)=\frac{i p}{\omega \mu(z)} G_{13}\left(z, z^{\prime}\right)+\frac{\delta\left(z-z^{\prime}\right)}{\omega \mu(z)},
\end{gathered}
$$

demonstrating in particular that the longitudinal component $G_{33}\left(z, z^{\prime}\right)$ is divergent at $z=z^{\prime}$, due to the $\delta$ function in the last term. Also, Eq. (A11) contains exactly the same operator as in Eq. (A3), and therefore

$$
G_{13}\left(z, z^{\prime}\right)=-\frac{i p}{\omega \mu\left(z^{\prime}\right)} G_{11}\left(z, z^{\prime}\right) .
$$

Using the reciprocity Eq. (A2) and Eqs. (A4), (A12), and (A14), we further obtain

$$
G_{12}\left(z, z^{\prime}\right)=G_{21}\left(z^{\prime}, z\right)=-\frac{1}{\omega \mu\left(z^{\prime}\right)} \partial_{z^{\prime}} G_{11}\left(z, z^{\prime}\right),
$$

$$
G_{32}\left(z, z^{\prime}\right)=G_{23}^{\dagger}\left(z^{\prime}, z\right)=-\frac{i p}{\omega^{2} \mu(z) \mu\left(z^{\prime}\right)} \partial_{z^{\prime}} G_{11}\left(z, z^{\prime}\right),
$$


and therefore

$$
\begin{aligned}
G_{22}\left(z, z^{\prime}\right) & =-\frac{1}{\omega \mu(z)} \partial_{z} G_{12}\left(z, z^{\prime}\right)+\frac{\delta\left(z-z^{\prime}\right)}{\omega \mu(z)} \\
& =\frac{1}{\omega^{2} \mu(z) \mu\left(z^{\prime}\right)} \partial_{z} \partial_{z^{\prime}} G_{11}\left(z, z^{\prime}\right)+\frac{\delta\left(z-z^{\prime}\right)}{\omega \mu(z)} .
\end{aligned}
$$

Finally, we find from Eq. (A5):

$$
G_{13}\left(z, z^{\prime}\right)=G_{31}^{\dagger}\left(z^{\prime}, z\right)=-\frac{i p}{\omega \mu\left(z^{\prime}\right)} G_{11}\left(z, z^{\prime}\right),
$$

in agreement with Eq. (A14). Collecting all this information about the GF components, we find a compact expression for the full dyadic GF:

$$
\begin{aligned}
\hat{\mathcal{G}}_{p}\left(z, z^{\prime}\right)= & \hat{O}_{p}(z ; \omega) \otimes \hat{O}_{-p}\left(z^{\prime} ; \omega\right) G_{11}\left(z, z^{\prime}\right) \\
& +\frac{\hat{\mathbf{1}}_{2}+\hat{\mathbf{1}}_{3}}{\omega \mu(z)} \delta\left(z-z^{\prime}\right),
\end{aligned}
$$

where $\hat{\mathbf{1}}_{j}$ are $3 \times 3$ matrices with elements $\left(\hat{\mathbf{1}}_{j}\right)_{i i^{\prime}}=\delta_{i i^{\prime}} \delta_{i j}$ and $\hat{O}_{p}$ is a vector operator defined as

$$
\hat{O}_{p}(z ; \omega)=\left(\begin{array}{c}
1 \\
-\frac{1}{\omega \mu(z)} \partial_{z} \\
\frac{i p}{\omega \mu(z)}
\end{array}\right) .
$$

Solving Eq. (A3) with outgoing boundary conditions and studying the pole structure and the cuts of the GF in the complex $\omega$ plane, as done in Appendix B for a general planar system, allows us to find the ML expansion for $G_{11}$ :

$$
G_{11}(z, z)=\sum_{n} \frac{E_{n}(z) E_{n}\left(z^{\prime}\right)}{\omega-\omega_{n}},
$$

and also for

$$
g\left(z, z^{\prime}\right)=\frac{G_{11}(z, z)}{\omega}=\sum_{n} \frac{E_{n}(z) E_{n}\left(z^{\prime}\right)}{\omega_{n}\left(\omega-\omega_{n}\right)},
$$

where $E_{n}(z)$ is the only nonvanishing component (directed along $y$ ) of the electric field of the RS $n$, which satisfies

$$
\hat{U}\left(\mu ; \omega_{n}\right) E_{n}(z)=0,
$$

or $E_{n}(z)$ is the wave function of the cut pole (see Appendix B for details). Obviously, $E_{n}(z)$ depends on $p^{2}$, as does the operator $\hat{U}$, and thus $E_{n}(z)$ is not sensitive to a change of sign of $p$, so that $E_{n}^{\dagger}(z)=E_{n}(z)$.

Comparing Eqs. (A21) and (A22) and substituting Eq. (A22) into Eq. (A3), we obtain with the help of Eq. (A23) a closure relation

$$
\varepsilon(z) \sum_{n} E_{n}(z) E_{n}\left(z^{\prime}\right)=\delta\left(z-z^{\prime}\right)
$$

and a sum rule

$$
\sum_{n} \frac{E_{n}(z) E_{n}\left(z^{\prime}\right)}{\omega_{n}}=0
$$

Using Eqs. (A21) and (A25), we obtain from Eq. (A19) the ML expansion (18) of the full dyadic GF, in which

$$
\mathcal{F}_{n}(z ; p)=\hat{O}_{p}\left(z ; \omega_{n}\right) E_{n}(z)
$$

Note that, in general, one also needs to include in Eq. (18) for completeness longitudinal static modes, in order to take into account the effect of the $\omega=0$ pole of the GF.

\section{APPENDIX B: MITTAG-LEFFLER EXPANSION OF THE GF OF A GENERAL HOMOGENEOUS PLANAR SYSTEM}

Let us now consider the scalar GF $g\left(z, z^{\prime}\right)$ of a general homogeneous system, described by arbitrary functions $\varepsilon(z)$ and $\mu(z)$, and derive its ML expansion given by Eq. (A22). This GF satisfies an equation

$$
\hat{U}_{k^{2}}(z) g\left(z, z^{\prime}\right)=\mu(z) \delta\left(z-z^{\prime}\right),
$$

where the operator $\hat{U}_{k^{2}}(z)$ is given by

$$
\hat{U}_{k^{2}}(z)=\mu(z) \partial_{z} \frac{1}{\mu(z)} \partial_{z}+k^{2} \varepsilon(z) \mu(z)+p^{2}[\varepsilon(z) \mu(z)-1],
$$

in accordance with its definition (A6), now written in terms of $k^{2}=\omega^{2}-p^{2}$.

To simplify the derivation, we assume that $\varepsilon(z)=\mu(z)=$ 1 outside the system $(|z|>a)$. In this case, Eq. (B1) outside the system takes the form

$$
\left(\partial_{z}^{2}+k^{2}\right) g\left(z, z^{\prime}\right)=\delta\left(z-z^{\prime}\right)
$$

with $k$ being the normal component of the wave number in the vacuum. Applying the outgoing wave boundary conditions, we find that

$$
g\left(z, z^{\prime}\right)=g\left( \pm a, z^{\prime}\right) e^{ \pm i k z} \text { for }|z|>a \text { and }\left|z^{\prime}\right|<a,
$$

where $+(-)$ refers to $z>a(z<-a)$. The outgoing wave boundary conditions for solving Eq. (B1) can therefore be written in the following way:

$$
\left.\left(\partial_{z} \mp i k\right) g\left(z, z^{\prime}\right)\right|_{z= \pm a}=0,
$$

explicitly showing that $g\left(z, z^{\prime}\right)$ is an analytic function of $k$. Having a countable number of simple poles in the complex $k$ plane, which are at the RS wave numbers $k_{n}=\sqrt{\omega_{n}^{2}-p^{2}}$, and are vanishing at $k \rightarrow \infty$, the $\mathrm{GF} g\left(z, z^{\prime}\right)$ can be written as

$$
g\left(z, z^{\prime}\right)=\sum_{n} \frac{R_{n}\left(z, z^{\prime}\right)}{k-k_{n}},
$$

using the Mittag-Leffler theorem [52]. To find an explicit form of the residue $R_{n}\left(z, z^{\prime}\right)$ we use Maxwell's wave equation without sources

$$
\hat{U}_{k_{n}^{2}}(z) E_{n}(z)=0,
$$

determining the RS wave functions $E_{n}(z)$, as well as the one with a source term [28,34],

$$
\hat{U}_{k^{2}}(z) E(z ; k)=\mu(z)\left(k-k_{n}\right) \sigma_{n}(z),
$$

determining its analytic continuation $E(z ; k)$ in the complex $k$ plane about the point $k_{n}$, such that $E\left(z ; k_{n}\right)=E_{n}(z)$. The source $\sigma_{n}(z)$ can be any function vanishing outside the system and normalized in such a way that

$$
\int_{-a}^{a} E_{n}(z) \sigma_{n}(z) d z=k_{n}
$$


In optical systems with degenerate RSs (e.g., due to symmetry), such that $k_{n}=k_{m}$ for $m \neq n, \sigma_{n}(z)$ is chosen in such a way that $\int_{-a}^{a} E_{m}(z) \sigma_{n}(z) d z=k_{n} \delta_{n m}$.

Solving Eq. (B8) with the help of the GF $g\left(z, z^{\prime}\right)$ and using its ML expansion (B6), we find

$$
E(z ; k)=\sum_{n^{\prime}} \frac{k-k_{n}}{k-k_{n^{\prime}}} \int_{-a}^{a} R_{n^{\prime}}\left(z, z^{\prime}\right) \sigma_{n}\left(z^{\prime}\right) d z^{\prime}
$$

Then taking the limit $k \rightarrow k_{n}$, Eq. (B10) becomes

$$
E_{n}(z)=\int_{-a}^{a} R_{n}\left(z, z^{\prime}\right) \sigma_{n}\left(z^{\prime}\right) d z^{\prime},
$$

which can be written, after combining it with Eq. (B9), as

$$
\int_{-a}^{a}\left[\frac{E_{n}(z) E_{n}\left(z^{\prime}\right)}{k_{n}}-R_{n}\left(z, z^{\prime}\right)\right] \sigma_{n}\left(z^{\prime}\right) d z^{\prime}=0
$$

The last equation must be satisfied for any normalized $\sigma_{n}(z)$, suited for generating the analytic continuation. Clearly, such a function is not unique, therefore, the integrand in the last equation should be vanishing, which gives $R_{n}\left(z, z^{\prime}\right)=$ $E_{n}(z) E_{n}\left(z^{\prime}\right) / k_{n}$ and results in the following series for the GF:

$$
g\left(z, z^{\prime}\right)=\sum_{n} \frac{E_{n}(z) E_{n}\left(z^{\prime}\right)}{k_{n}\left(k-k_{n}\right)} .
$$

We now find the normalization of $E_{n}(z)$ which is determined by the ML form Eq. (B13), which in turn follows from the normalization of the source Eq. (B9). We therefore use it again, substituting $\sigma_{n}(z)$ from Eq. (B8) into Eq. (B9) and subtracting a similar integral vanishing due to Eq. (B7):

$$
\begin{aligned}
\left(k-k_{n}\right) k_{n} & =\int_{-a}^{a} \frac{1}{\mu} E_{n} \hat{U}_{k^{2}} E d z-\int_{-a}^{a} \frac{1}{\mu} E \hat{U}_{k_{n}^{2}} E_{n} d z \\
& =\left[\frac{E_{n}}{\mu} \partial_{z} E-\frac{E}{\mu} \partial_{z} E_{n}\right]_{-a}^{a}+\left(k^{2}-k_{n}^{2}\right) \int_{-a}^{a} E_{n} E \varepsilon d z,
\end{aligned}
$$

where the first term in the second line is obtained integrating by parts. Finally, using the outgoing wave boundary conditions for $E$ and $E_{n}$,

$$
\begin{aligned}
& \left.\left(\frac{1}{\mu(z)} \partial_{z} \mp i k\right) E(z ; k)\right|_{z= \pm a}=0, \\
& \left.\left(\frac{1}{\mu(z)} \partial_{z} \mp i k_{n}\right) E_{n}(z)\right|_{z= \pm a}=0,
\end{aligned}
$$

similar to Eq. (B5), we arrive, after taking again the limit $k \rightarrow$ $k_{n}$, at the normalization condition for the RS wave function:

$$
2 \int_{-a}^{a} E_{n}^{2}(z) \varepsilon(z) d z-\frac{E_{n}^{2}(a)+E_{n}^{2}(-a)}{i k_{n}}=1,
$$

which is the same as the one provided in [18] without proof.

Note that Eq. (B16) is equivalent to the general normalization (20) used for the TE polarization. In fact, using Eq. (20) for $z_{1}=-a$ and $z_{2}=a$ and the fields replaced by their analytic continuations for the purpose of taking the frequency derivatives, we find (denoting here for brevity $i H$ with just $H$ )

$$
1=\int_{-a}^{a}\left[\varepsilon E^{2}+\mu\left(H_{x}^{2}-H_{z}^{2}\right)\right] d z-\left[E \partial_{\omega} H_{x}-H_{x} \partial_{\omega} E\right]_{-a}^{a}
$$

[see Eqs. (5), (A20), and (A26)]. Then, using

$$
H_{x}=-\frac{1}{\omega \mu} \partial_{z} E, \quad H_{z}=\frac{i p}{\omega \mu} E,
$$

and

$$
\left(\mu \partial_{z} \frac{1}{\mu} \partial_{z}+\omega^{2} \varepsilon \mu-p^{2}\right) E=0,
$$

valid for $k=k_{n}$, we find, integrating by parts,

$$
\begin{aligned}
& \int_{-a}^{a} \mu\left(H_{x}^{2}-H_{z}^{2}\right) d z \\
& =\frac{1}{\omega^{2}} \int_{-a}^{a} \frac{1}{\mu}\left[\left(\partial_{z} E\right)^{2}+p^{2} E^{2}\right] d z \\
& =\frac{\left[E \partial_{z} E\right]_{-a}^{a}}{\omega^{2}}+\frac{1}{\omega^{2}} \int_{-a}^{a} \frac{1}{\mu}\left[-E \mu \partial_{z} \frac{1}{\mu} \partial_{z} E+p^{2} E^{2}\right] d z \\
& =\frac{\left[E \partial_{z} E\right]_{-a}^{a}}{\omega^{2}}+\int_{-a}^{a} \varepsilon E^{2} d z .
\end{aligned}
$$

We then use the analytic form of the fields outside the system:

$$
E(z ; k)=A_{ \pm} e^{ \pm i k z}, \quad H_{x}(z ; k)=\mp \frac{i k}{\omega} E(z ; k),
$$

where, again, the upper (lower) sign corresponds to $z \geqslant a$ $(z \leqslant-a)$, and the amplitudes $A_{ \pm}$are also functions of $\omega$ or $k$. However, their frequency dependence does not contribute to the normalization since

$$
E \partial_{\omega} H_{x}-H_{x} \partial_{\omega} E=\mp E^{2} \partial_{\omega} \frac{i k}{\omega}=\mp \frac{p^{2}}{i k \omega^{2}} E^{2} .
$$

Collecting all the "surface" terms and differentiating the field outside the system, using the explicit form of $E$ given by Eq. (B21), we obtain

$$
\begin{aligned}
- & {\left[E \partial_{\omega} H_{x}-H_{x} \partial_{\omega} E\right]_{-a}^{a}+\frac{\left[E \partial_{z} E\right]_{-a}^{a}}{\omega^{2}} } \\
& =\left(\frac{i p^{2}}{k \omega^{2}}+\frac{i k}{\omega^{2}}\right)\left[E^{2}(a)+E^{2}(-a)\right] \\
& =-\frac{E^{2}(a)+E^{2}(-a)}{i k},
\end{aligned}
$$

which proves that Eq. (20) results in the normalization given by Eq. (B16).

Equation (B13) is the ML series of the GF in $k$ representation. However, the RSE formulated in Sec. II requires a ML form of the GF in the $\omega$ representation. Being treated as a function of frequency $\omega$, the GF has simple poles due to the RSs at $\omega=\omega_{n}$ (where $\omega_{n}^{2}=k_{n}^{2}+p^{2}$ ) which are distributed in the complex $\omega$ plane symmetrically with respect to the imaginary axis (see Fig. 8). The residues of the GF at these poles are given by

$$
\begin{aligned}
\lim _{\omega \rightarrow \omega_{n}} g\left(z, z^{\prime}\right)\left(\omega-\omega_{n}\right) & =\left.\frac{\partial \omega}{\partial k}\right|_{k_{n}} \lim _{k \rightarrow k_{n}} g\left(z, z^{\prime}\right)\left(k-k_{n}\right) \\
& =\frac{k_{n}}{\omega_{n}} R_{n}\left(z, z^{\prime}\right),
\end{aligned}
$$

where $R_{n}\left(z, z^{\prime}\right)$ is found earlier [see Eq. (B13)].

Apart from these poles, the GF $g\left(z, z^{\prime}\right)$ is analytic in the complex $k$ plane, as shown above. However, in the complex 


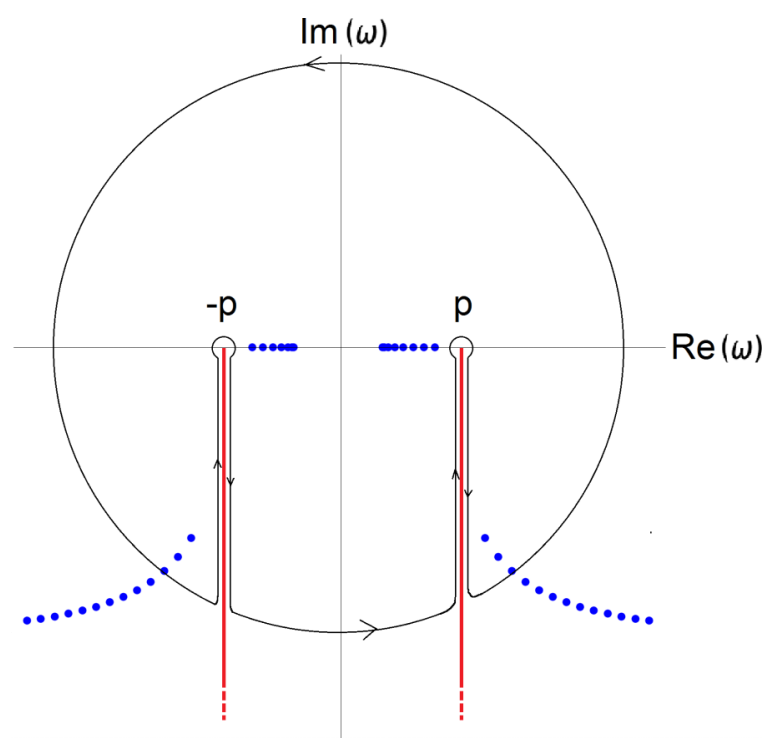

FIG. 8. Poles (blue dots) and cuts (red lines) of the GF in the complex $\omega$ plane, as well the contour of integration leading to the ML expansion (B26), which includes the cut contributions displayed.

$\omega$ plane $g\left(z, z^{\prime}\right)$ has branch cuts, owing to the link between $k$ and $\omega$,

$$
k=\sqrt{\omega^{2}-p^{2}},
$$

with the branch points at $\omega= \pm p$. Therefore, applying the ML theorem in the frequency plane results instead in

$$
g\left(z, z^{\prime}\right)=\sum_{n} \frac{E_{n}(z) E_{n}\left(z^{\prime}\right)}{\omega_{n}\left(\omega-\omega_{n}\right)}+I_{1}\left(z, z^{\prime} ; \omega\right)+I_{2}\left(z, z^{\prime} ; \omega\right),
$$

where the sum includes only the RSs on the selected Riemann sheet, which in our case includes all the WG and FP modes but does not contain anti-WG modes, unlike the series in Eq. (B13). Integrals

$$
I_{1,2}\left(z, z^{\prime} ; \omega\right)=-\frac{1}{2 \pi i} \int_{ \pm p}^{ \pm p-i \infty} \frac{\Delta g\left(z, z^{\prime} ; \omega^{\prime}\right)}{\omega-\omega^{\prime}} d \omega^{\prime}
$$

describe the contribution of the cuts, which are chosen as vertical straight lines in the complex $\omega$ plane going from the branch points $\pm p$ down to $-i \infty$ (see Fig. 8). According to [15], this choice of the cuts almost minimizes their contribution to the GF. The jump of the GF value across the cut is given by the function

$$
\Delta g\left(z, z^{\prime} ; \omega\right)=g_{k}\left(z, z^{\prime}\right)-g_{-k}\left(z, z^{\prime}\right),
$$

where we have added index $k$ for convenience, in order to emphasize the fact that $k$ is changing to $-k$ when going through the cut with an infinitesimal change of $\omega$.

The jump of the GF described by Eq. (B28) can be evaluated in the general case by using the explicit form of the GF,

$$
g\left(z, z^{\prime}\right)=\frac{E_{L}\left(z_{<}\right) E_{R}\left(z_{>}\right)}{E_{L} \dot{E}_{R}-\dot{E}_{L} E_{R}},
$$

in terms of the "left" and "right" functions $E_{L}(z)$ and $E_{R}(z)$, respectively. These are solutions of the homogeneous wave equation (B19) and the left or right outgoing boundary condition. More explicitly, they are given by

$$
E_{L}(z)= \begin{cases}e^{-i k z}, & z<-a \\ B_{+} f_{+}(z)+B_{-} f_{-}(z), & |z|<a\end{cases}
$$

and

$$
E_{R}(z)= \begin{cases}C_{+} f_{+}(z)+C_{-} f_{-}(z), & |z|<a \\ e^{i k z}, & z>a\end{cases}
$$

in terms of $f_{+}(z ; \omega)$ and $f_{-}(z ; \omega)$, two arbitrary linearly independent solutions of Eq. (B19) within the slab. While these functions (depending on $\omega$ ) do not change when changing the sign of $k$, the coefficients $B_{ \pm}$and $C_{ \pm}$do modify, leading to nonvanishing contributions to the jump $\Delta g$ of the GF across the cuts. Here, in Eq. (B29), $z_{<}=\min \left(z, z^{\prime}\right)$ and $z_{>}=$ $\max \left(z, z^{\prime}\right)$, and the derivative

$$
\dot{E}(z) \equiv \frac{1}{\mu(z)} \partial_{z} E(z)
$$

is introduced for convenience.

Now, choosing the functions $f_{ \pm}(z)$ in such a way that

$$
\begin{aligned}
& f_{+}(a) f_{-}(a)+f_{+}(-a) f_{-}(-a)=0, \\
& \dot{f}_{+}(a) \dot{f}_{-}(a)+\dot{f}_{+}(-a) \dot{f}_{-}(-a)=0, \\
& f_{+}(-a) \dot{f}_{+}(a)+f_{+}(a) \dot{f}_{+}(-a)=0, \\
& f_{-}(-a) \dot{f}_{-}(a)+f_{-}(a) \dot{f}_{-}(-a)=0,
\end{aligned}
$$

which can always be fulfilled, for any profiles of $\varepsilon(r)$ and $\mu(r)$. We obtain, after simple algebra, a convenient form of the cut integrand

$$
-\frac{1}{2 \pi i} \Delta g\left(z, z^{\prime} ; \omega\right)=\sum_{s= \pm} \sigma_{s} f_{s}(z) f_{s}\left(z^{\prime}\right)
$$

where

$$
\sigma_{s}(\omega)=\frac{1}{\pi} \frac{k}{\dot{f}_{s}^{2}(a)+\dot{f}_{s}^{2}(-a)+k^{2}\left[f_{s}^{2}(a)+f_{s}^{2}(-a)\right]} .
$$

This form allows us to include the contribution of the cuts on equal footing with the RSs, treating the cuts as continua of poles of the GF:

$$
\begin{aligned}
g\left(z, z^{\prime}\right)= & \sum_{n} \frac{E_{n}(z) E_{n}\left(z^{\prime}\right)}{\omega_{n}\left(\omega-\omega_{n}\right)} \\
& +\sum_{s= \pm} \sum_{s^{\prime}= \pm} \int_{s^{\prime} p}^{s^{\prime} p-i \infty} \frac{E_{s}\left(z ; \omega^{\prime}\right) E_{s}\left(z^{\prime} ; \omega^{\prime}\right)}{\omega^{\prime}\left(\omega-\omega^{\prime}\right)} d \omega^{\prime} \\
\equiv & \sum_{n} \frac{E_{n}(z) E_{n}\left(z^{\prime}\right)}{\omega_{n}\left(\omega-\omega_{n}\right)}
\end{aligned}
$$

where

$$
E_{s}(z ; \omega)=\sqrt{\omega \sigma_{s}(\omega)} f_{s}(z ; \omega) .
$$

Note that in Eqs. (B38), (B39), and (B40), we have added $\omega$ to the arguments of $E_{s}, \sigma_{s}$, and $f_{s}$, earlier omitted for brevity of notations, in order to emphasize now their frequency dependence. 


\section{APPENDIX C: HOMOGENEOUS SLAB WITH CONSTANT PERMITTIVITY AND PERMEABILITY}

Consider a dielectric slab in a vacuum, having thickness $2 a$ and constant permittivity and permeability. Their profiles in space are described by

$$
\begin{gathered}
\varepsilon(z)=1+(\epsilon-1) \Theta(a-|z|), \\
\mu(z)=1+(\mu-1) \Theta(a-|z|) .
\end{gathered}
$$

Within the slab $\left(|z|,\left|z^{\prime}\right| \leqslant a\right)$ the GF has the form

$$
g\left(z, z^{\prime}\right)=-\frac{\mu}{2 i q} \frac{\varphi\left(z_{<}\right) \varphi\left(-z_{>}\right)}{1-\xi^{2}} ;
$$

see Eq. (B29) in which, due to the mirror symmetry, the left and right solutions are given by the same function $E_{L}(z)=$ $E_{R}(-z)=\varphi(z)$, with

$$
\begin{gathered}
\varphi(z)=e^{i q z}+\xi e^{-i q z}, \\
\xi=\frac{1+\eta}{1-\eta} e^{-2 i q a}, \quad \eta=\frac{\mu k}{q}, \\
q^{2}=\epsilon \mu \omega^{2}-p^{2}, \quad k^{2}=\omega^{2}-p^{2} .
\end{gathered}
$$

Clearly, the GF has poles at $\xi= \pm 1$, determining the secular equation for the RS frequencies $\omega_{n}$ :

$$
\left(q_{n}+\mu k_{n}\right) e^{-i q_{n} a}=(-1)^{n}\left(q_{n}-\mu k_{n}\right) e^{i q_{n} a} .
$$

The RS wave functions, which are the solutions of Eq. (B7), are given by

$$
E_{n}(z)= \begin{cases}A_{n} e^{i k_{n} z}, & z>a \\ B_{n}\left(e^{i q_{n} z}+(-1)^{n} e^{-i q_{n} z}\right), & |z| \leqslant a \\ (-1)^{n} A_{n} e^{-i k_{n} z}, & z<-a\end{cases}
$$

with the continuity condition $A_{n}=B_{n}\left(e^{i q_{n} a}+\right.$ $\left.(-1)^{n} e^{-i q_{n} a}\right) e^{-i k_{n} a}$. The eigenvalues $k_{n}$ and $q_{n}$ are related to the eigenfrequency $\omega_{n}$ via Eq. (C6), and the normalization constants $B_{n}$ found from Eq. (B16) have the following explicit form:

$$
B_{n}^{-2}=8(-1)^{n}\left[\epsilon a-\frac{i p^{2}(\epsilon \mu-1)}{k_{n}\left(q_{n}^{2}-\mu^{2} k_{n}^{2}\right)}\right] .
$$

For the cuts of the GF in the complex $\omega$ plane, the functions satisfying Eqs. (B33)-(B36) are given by

$$
f_{ \pm}(z)=e^{i q z} \pm e^{-i q z}
$$

within the slab $|z| \leqslant a$. They possess a definitive parity $(s=$ \pm 1 ) due to the mirror symmetry of the system, and the same form as the RS wave functions (C8). According to Eq. (B38), the cut density functions are given by

$$
\sigma_{ \pm}(\omega)=\frac{1}{4 \pi} \frac{\mu^{2} k}{\left(\mu^{2} k^{2}-q^{2}\right) \cos (2 q a) \pm\left(\mu^{2} k^{2}+q^{2}\right)} .
$$

\section{GF in $k$ representation}

Using the Newton-Raphson method, we have solved the secular equation (C7) and found all the RS wave numbers in a selected frequency range (within a circle of radius $\omega_{\max }$ in the
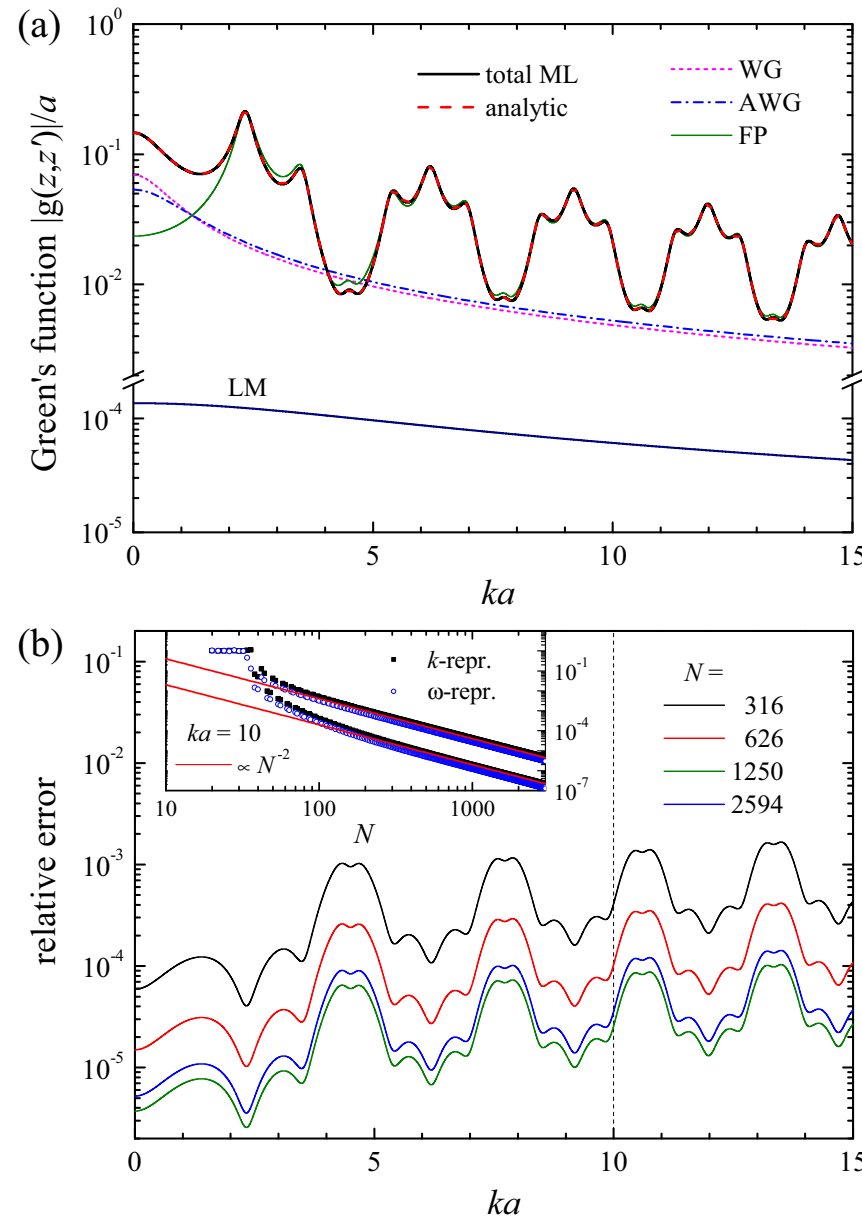

FIG. 9. (a) Green's function $g\left(z, z^{\prime}\right)$ of a homogeneous dielectric slab in vacuum, with $\epsilon=6$, calculated for $p a=5, z=a / 2$, and $z^{\prime}=$ $-a / 2$ as a function $k$, the normal component of the wave number in the vacuum. Partial contributions of WG, anti-WG (AWG), FP modes, and a leaky mode (LM) are shown along with the sum of all the contributions and the analytic values of the GF. (b) Relative error of the GF calculated via Eq. (B13) as compared to its analytic values Eq. (C3), for different number of modes $N$ included in the ML series. The inset shows the relative error for $k a=10$ [marked by vertical dashed lines in Figs. 9(b) and 10(b)] as a function of the basis size $N$.

complex frequency plane). The RSs include four categories of modes: WG modes, anti-WG modes, FP modes, and a leaky mode (LM). The WG and anti-WG modes are present only if $p \neq 0$. We then use the ML expansion (B13) in the $k$ representation which includes all types of modes in the summation and compare it with the analytic GF given by Eq. (C3)

Results for a slab with $\epsilon=6$ and $\mu=1$ are shown in Fig. 9 for $z=-z^{\prime}=a / 2$. We see that all partial contributions to the GF due to each type of mode is nonvanishing, including that of the LM which has $\operatorname{Re} \omega_{n}=0$ and which is discussed in detail in $[18,32]$. Summing up all the contributions to the ML series (B13) results in values of the GF approaching its analytic form (C3). By increasing $\omega_{\max }$, we increase the number of RSs $N$ included in the series (B13), in this way making the ML representation more and more accurate [see 

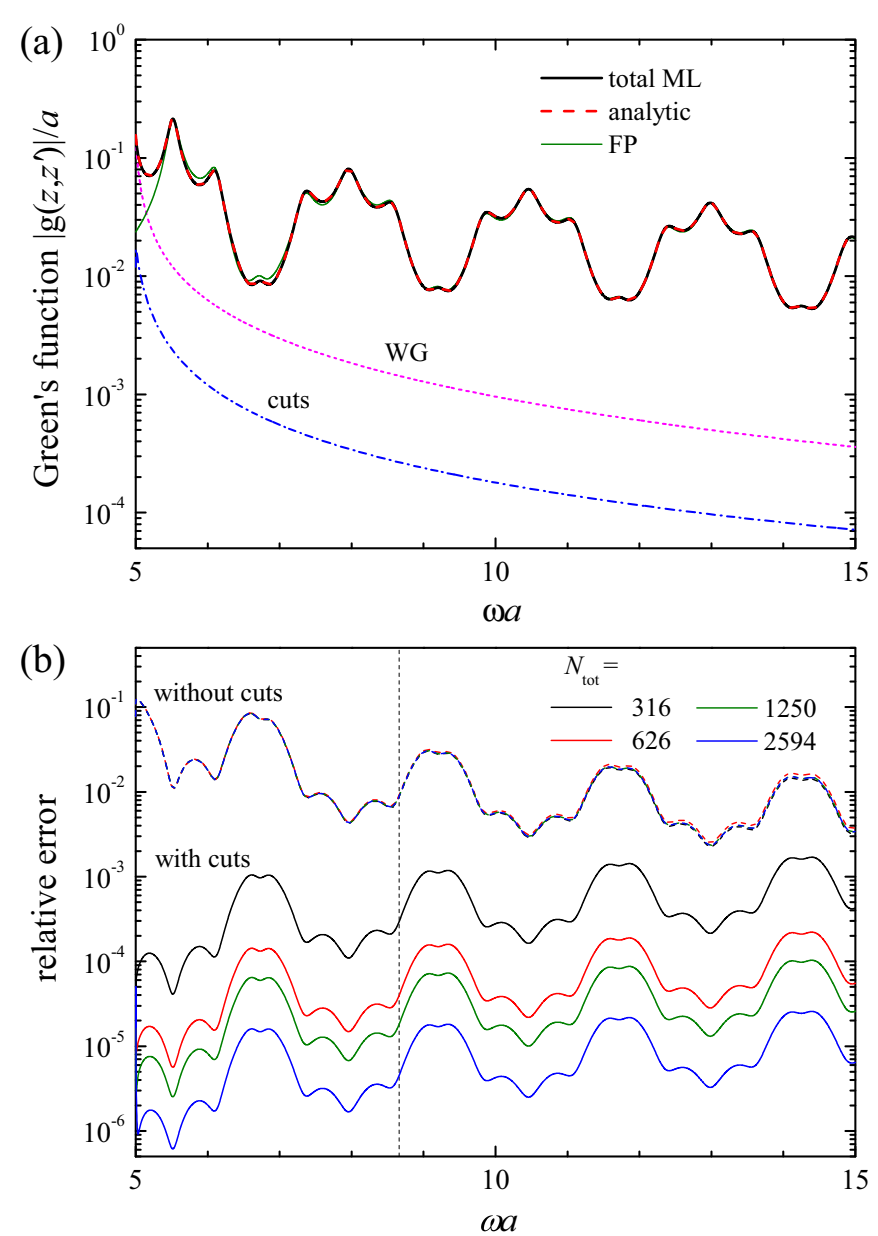

FIG. 10. (a) As Fig. 9(a) but in the $\omega$ representation, in which the AWG modes and the LM do not contribute to the ML expansion (B39), but there is instead an additional component due to the cuts. (b) Relative error of the GF calculated via Eq. (B39) as compared to its analytic values (C3), for different number of modes $N$ included in the ML series, with and without contribution of the cuts. The dashed line indicates where the value of $\omega a$ is equivalent to $k a=10$.

Fig. 9(b)]. The inset demonstrates the convergence of the ML series to the exact solution, with the error scaling as $1 / N^{2}$.

\section{GF in $\omega$ representation}

The GF can also be represented as a function of frequency. However, the square root in Eq. (B25) causes branch cuts at $\omega= \pm p$, which separates the frequency plane into two Riemann sheets with modes split across both sheets. Only the modes found on one of the sheets are taken into account. This is chosen to be the "physical" sheet, on which the WG and FP modes are found, while anti-WG modes and the leaky mode turn out to be on the other, unphysical sheet and are thus excluded from the ML expansion (B39). Figure 10 demonstrates a comparison, for the same parameters as in Fig. 9, of the ML expansion (B39) with the analytic solution (C3), again showing different contributions, including WG, FP modes, and the cuts. We see that the ML series in the $\omega$ representation again converges to the exact solution, provided that the cut
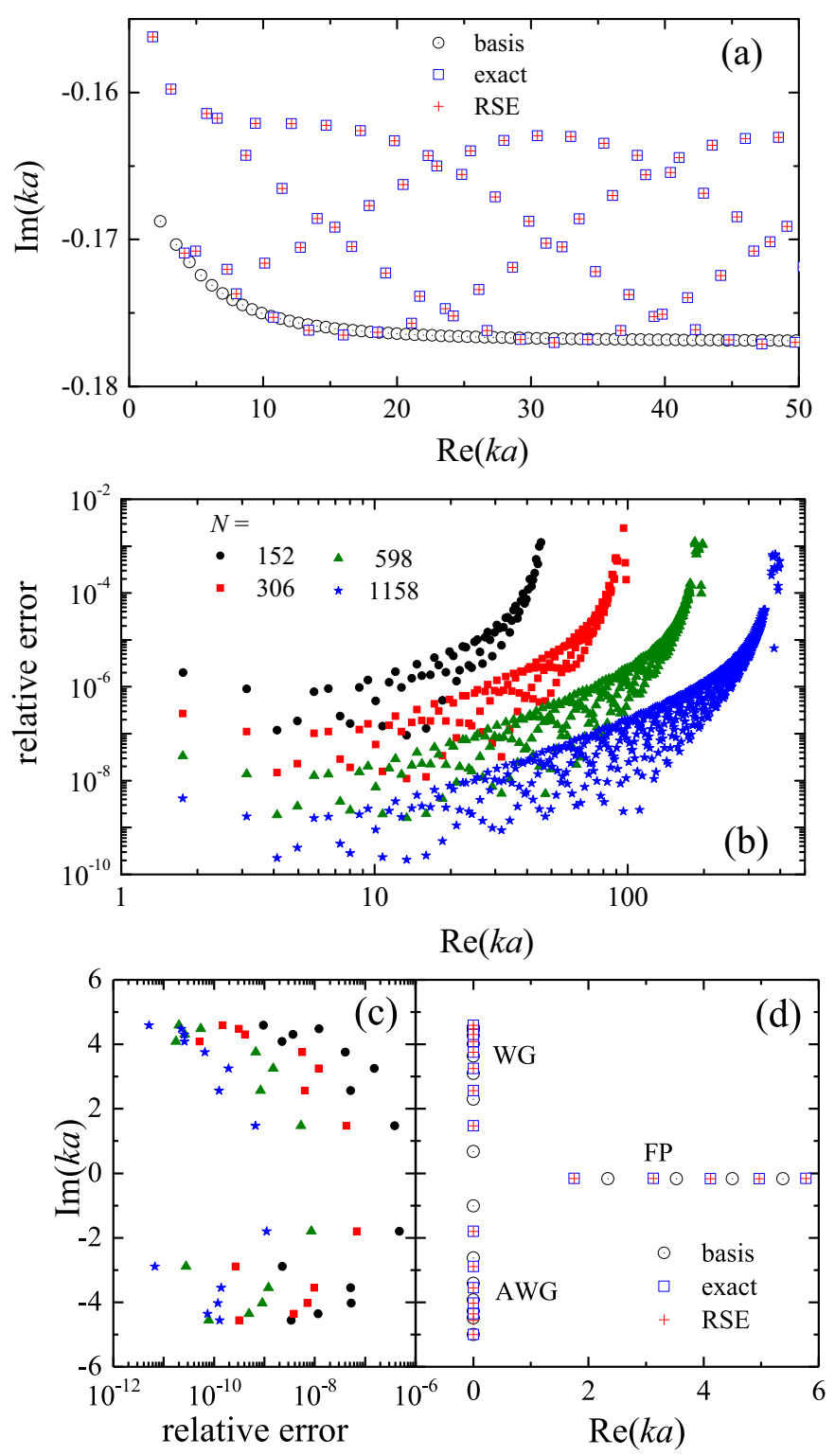

FIG. 11. (a) Wave numbers $k_{n}$ of the FP modes calculated for $p a=5$ using the RSE in the $k$ representation (red crosses) for the core-shell dielectric slab with $\epsilon=6, \Delta \epsilon=1$, and $b=a / 2$, along with the exact solution (blue squares) and the basis RSs (black circles with dots). (b) Relative error for the wave numbers of the FP modes compared to the exact solution, for different basis sizes as given. (c) Relative errors for WG and anti-WG modes, for the same basis sizes as in (b). (d) As (a) but with all WG and anti-WG modes, and the first few FP modes shown.

integrals are included. Note also that the convergence is very similar to that in the $k$ representation, as it is clear from the inset in Fig. 9(b). In fact, in both cases the error alternates between two different boundaries but nevertheless decreases with the basis size as $N^{-2}$.

\section{APPENDIX D: RSE FOR A HOMOGENEOUS CORE SLAB}

Here, we demonstrate how the RSE in the $k$ and $\omega$ representations is applied to a homogeneous perturbation. This is a special case of the PC-RSE which allows an exact analytic 


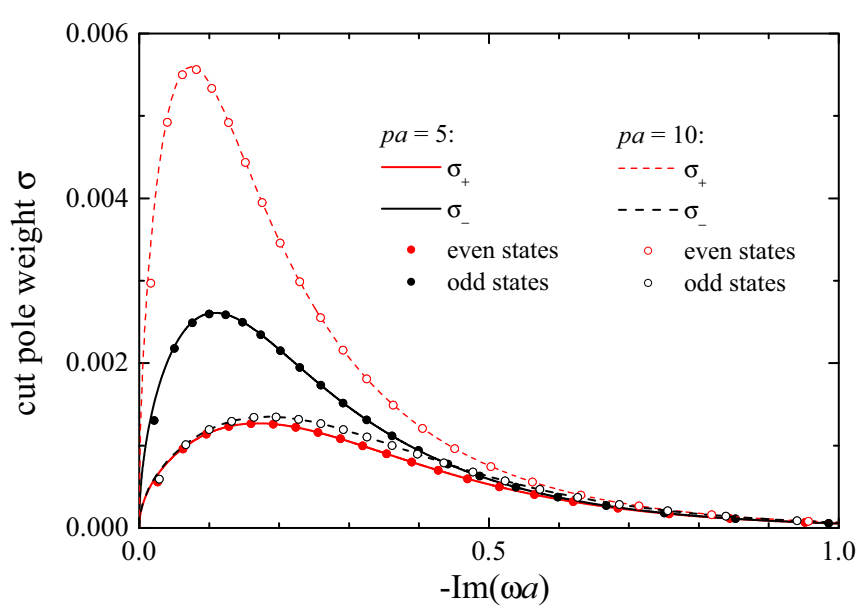

FIG. 12. Cut weights $\sigma_{ \pm}$(solid and dashed lines) as functions of the imaginary part of the frequency along the cut, for different values of $p$ as given, along with the discretized cut pole values $C_{v} / \Delta \omega_{v}$ (circles).

solution, but obviously lacking any periodic modulations. We use $\alpha=\Delta \epsilon$ and $\beta=0$ in Eq. (32). The perturbation of the permittivity thus has the form

$$
\Delta \varepsilon(z)=\Delta \epsilon \Theta(b-|z|) .
$$

The perturbed system presents a core-shell slab consisting of three homogeneous regions. The secular equation for this system has the following analytic form:

$$
\left(\gamma_{+} \lambda_{-}-\gamma_{-} \lambda_{+}\right) e^{i q_{1} b} \pm\left(\gamma_{+} \lambda_{+}-\gamma_{-} \lambda_{-}\right) e^{-i q_{1} b}=0,
$$

where $\quad \gamma_{ \pm}=(q \pm k) e^{\mp i q(a-b)}, \quad \lambda_{ \pm}=q \pm q_{1}, \quad$ and $\quad q_{1}=$ $\sqrt{\epsilon_{1} \omega^{2}-p^{2}}$ with $\epsilon_{1}=\epsilon+\Delta \epsilon$. The secular equation (C7) for the homogeneous slab can be restored by setting $b=a$ and $q=q_{1}$, or simply $b=0$.

\section{1. $\mathrm{RSE}$ in $k$ representation}

In the $k$ representation, the RSE equation for treating planar homogeneous systems is given by Eq. (22) of [18], which we write here as

$$
k \sum_{n^{\prime}}\left(\delta_{n n^{\prime}}+\mathcal{V}_{n n^{\prime}}\right) c_{n^{\prime}}=\sum_{n^{\prime}}\left(k_{n} \delta_{n n^{\prime}}-\frac{p^{2}}{k_{n}} \mathcal{V}_{n n^{\prime}}\right) c_{n^{\prime}},
$$

with the matrix elements $\mathcal{V}_{n n^{\prime}}=V_{n n^{\prime}}^{00}$ given by Eq. (33) for $g=$ $g^{\prime}=0$.

Its application to the perturbation given by Eq. (D1) is shown in Fig. 11, in comparison with the exact solution (D2) and the basis RSs of the homogenous slab. The quasiperiodic pattern of the wave numbers of the perturbed RSs seen in Fig. 11 is caused by the perturbation covering only the middle half of the slab, so that the original homogeneous slab of thickness $2 a$ is now split into three subsystems of thickness $a / 2, a$, and $a / 2$, each acting as a resonance cavity. The distance in frequency between the modes is given by a fundamental period of $\pi /(2 a \sqrt{\epsilon})$ as in the basis cavity, but the cavities between $-a$ and $-b$ and between $b$ and $a$ have caused additional quasiperiodicities, one of them having the period of $\pi /[(a-b) \sqrt{\epsilon}$, which for $b=a / 2$ is four times larger than the
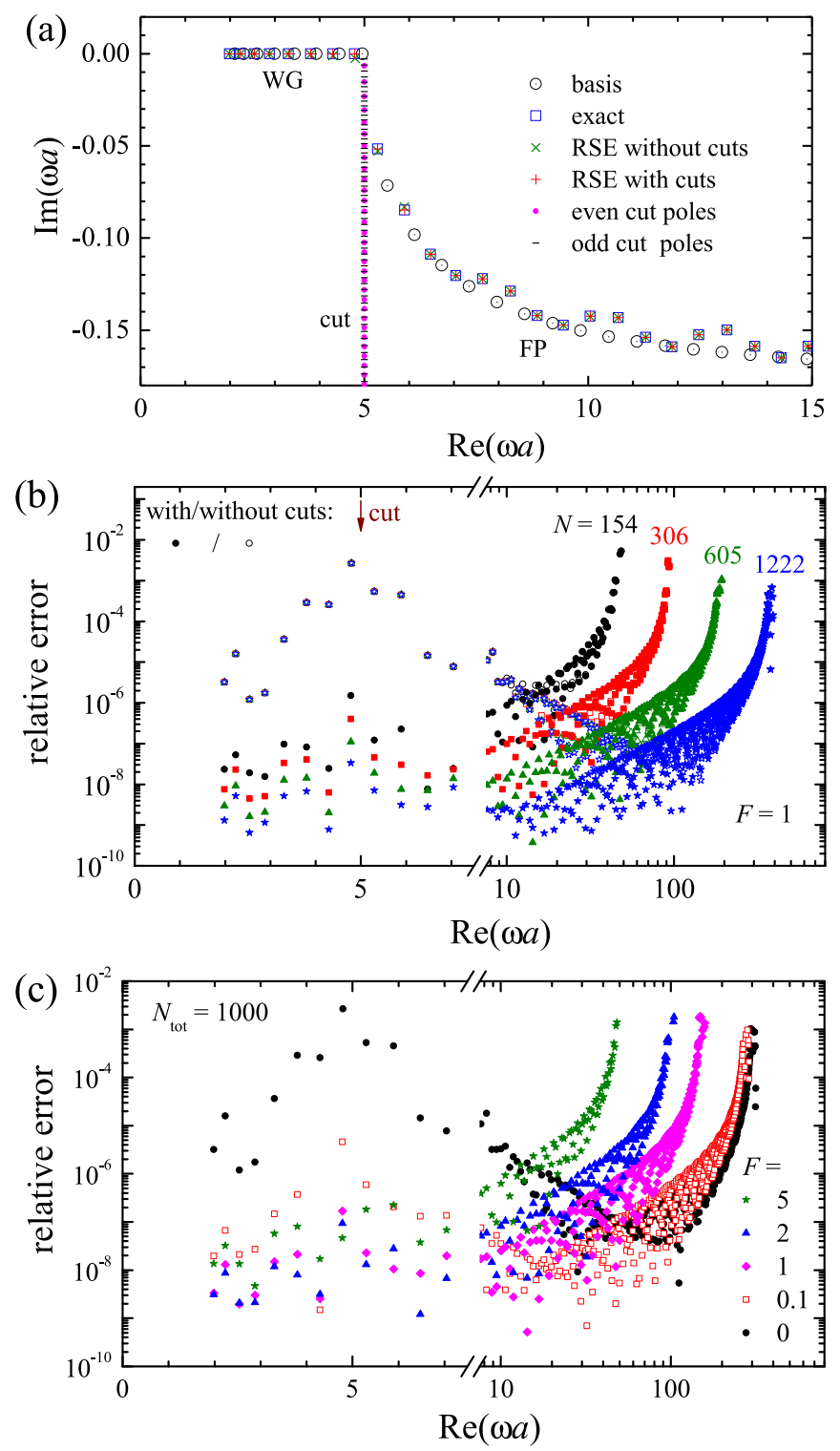

FIG. 13. (a) As Fig. 11 but in frequency representation, also showing even- and odd-parity cut modes of the homogeneous slab. (b) Relative errors compared to the exact solution (D2) of the RS frequencies calculated using the RSE, with and without cut modes in the basis and for different basis sizes as given. (c) Relative error of the RSE results calculated for a fixed basis size $N_{\text {tot }}=1000$ and different values of $F$, the ratio of the number of cut modes to the number of RSs in the basis.

fundamental period. Other cavities present in the system also contribute to the rich spectrum of RSs seen in Fig. 11.

Looking at the relative error shown in Figs. 11(b) and $11(\mathrm{c})$, we see that the RSE in the $k$ representation quickly converges to the exact solution. The relative error scales as $1 / N^{3}$, which is typical for effective $1 \mathrm{D}$ systems (see $[14,18,27,28,31])$.

\section{RSE in $\omega$ representation}

The RSE equation in this case is given by the general formula (23) of the PC-RSE, but since this is a homogeneous 

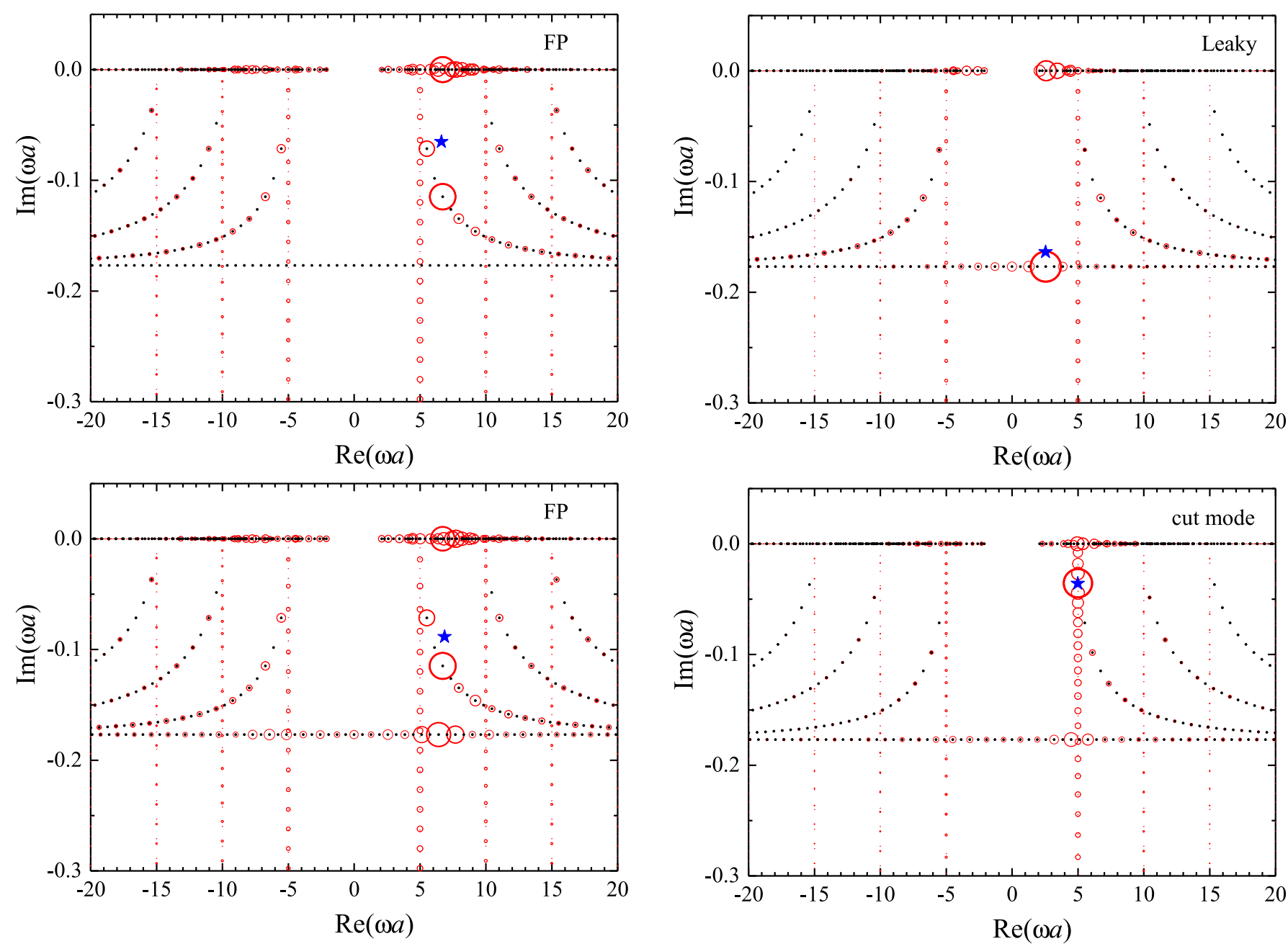

FIG. 14. As Fig. 7 but for two RSs originating from a degenerate pair of FP modes.

perturbation, there is no mixing of channels, so we use again $g=g^{\prime}=0$. Also, Eq. (23) includes the contribution of the cuts which need to be discretized, giving rise to cut modes to be used in the RSE on equal footing with the RSs.

The discretization of the cuts is done following the procedure described in $[14,15]$. For each parity $s$, the cut with the branch point at $\omega=p$ is divided into $N_{c}$ intervals bounded by $\left[\tilde{\omega}_{v}, \tilde{\omega}_{v+2}\right]$, where $v$ is even (odd) for $s=+(s=-)$, with a weight given by

$$
W_{s}=\int_{\tilde{\omega}_{v}}^{\tilde{\omega}_{v+2}} \sqrt{\left|\sigma_{s}(\omega)\right|} d \omega
$$

where $\sigma_{s}$ is given by Eq. (C11), $\tilde{\omega}_{1}=\tilde{\omega}_{2}=p$ and $\tilde{\omega}_{2 N_{c}+1}=$ $\tilde{\omega}_{2 N_{c}+2}=p-i \infty$. The cut is split into intervals in such a way that $W_{s}$ is the same for each interval (for the given parity $s$ ). Within each interval, an artificial cut mode is defined at the frequency $\omega_{\nu}$, given by

$$
\omega_{\nu}=\frac{1}{C_{\nu}} \int_{\tilde{\omega}_{\nu}}^{\tilde{\omega}_{v+2}} \sigma_{s}(\omega) \omega d \omega
$$

with $v=1,2, \ldots, 2 N_{c}$, where $s=(-1)^{v}$ and

$$
C_{v}=\int_{\tilde{\omega}_{v}}^{\tilde{\omega}_{v+2}} \sigma_{s}(\omega) d \omega .
$$

FIG. 15. As Fig. 7 but for a RS coming from a nondegenerate $m=0$ leaky mode (top) and for a perturbed cut mode (bottom).

Applying the same discretization to the other cut with the branch point at $\omega=-p$ and extending the $v$ numbers to negative integers, the ML expansion (B39) takes the form

$$
g\left(z, z^{\prime}\right) \approx \sum_{\bar{n}} \frac{E_{\bar{n}}(z) E_{\bar{n}}\left(z^{\prime}\right)}{\omega_{\bar{n}}\left(\omega-\omega_{\bar{n}}\right)},
$$

where

$$
\bar{n}= \begin{cases}n & \text { for RSs } \\ v & \text { for cut modes }\end{cases}
$$

and

$$
E_{\bar{n}}(z)=B_{\bar{n}}\left[e^{i q_{\bar{n}} z}+(-1)^{\bar{n}} e^{-i q_{\bar{n}} z}\right], \quad|z| \leqslant a
$$

with the normalization constant $B_{n}$ for the RSs given by Eq. (C9) and for the cut modes by

$$
B_{v}=\sqrt{\omega_{\nu} C_{\nu}}
$$

where $C_{v}$ is defined in Eq. (D6). Using Eq. (D6), we graphically show in Fig. 12 how these artificial cut modes compensate for the cut, comparing $\sigma_{v}(\omega)$ with $C_{\nu} / \Delta \omega_{\nu}$, where $\Delta \omega_{\nu}=\tilde{\omega}_{\nu+2}-\tilde{\omega}_{\nu}$ is the interval of integration.

With these modes added to the basis, the RSE can now be performed in the $\omega$ representation using Eq. (28) with the 

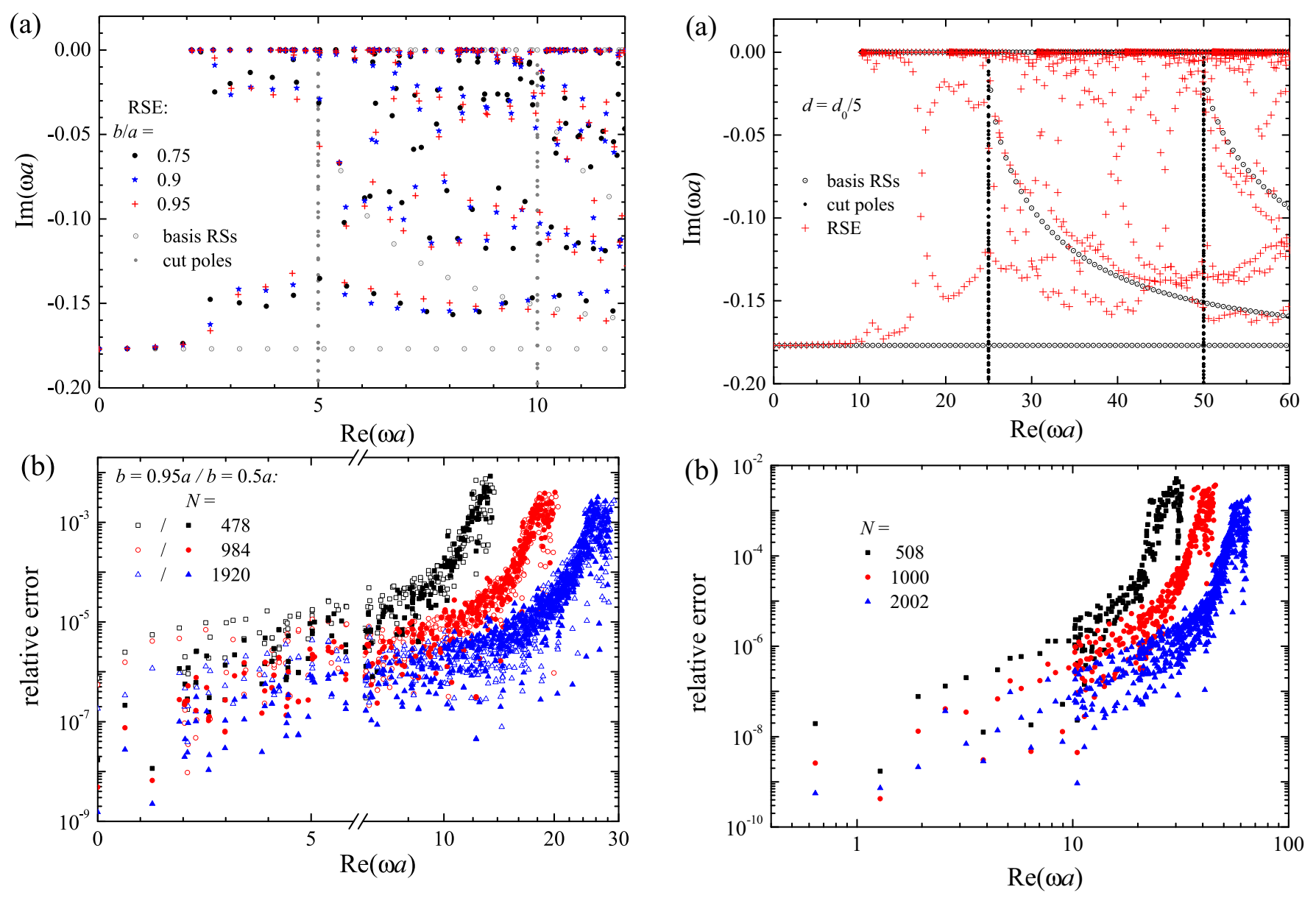

FIG. 16. (a) RS frequencies of a PC slab with $\epsilon=6, \alpha=0$, $\beta=1, d=2 \pi a / 5$, and $p=0$, calculated for $F=1$ and $M=5$ via the PC-RSE for different values of $b$ as given. Unperturbed RSs and cut modes are also shown (gray circles with dots and gray dots, respectively). (b) Relative error of the PC-RSE compared to the SMM result, taking the latter as "exact," calculated for different values of $b$ and different basis sizes $N$ as given.

index $g$ dropped:

$$
\omega \sum_{\bar{n}^{\prime}}\left(\delta_{\bar{n} \bar{n}^{\prime}}+\mathcal{V}_{\bar{n} \bar{n}^{\prime}}\right) c_{\bar{n}^{\prime}}=\omega_{\bar{n}} c_{\bar{n}} .
$$

Figure 13(a) shows the RS frequencies calculated using the RSE equation (D11), with and without cut modes in the basis. The unperturbed RSs and even- and odd-parity cut modes of the basis are also shown. The distribution of perturbed RSs repeats the oscillatory pattern seen in Fig. 11(a) and discussed above. The frequencies of RSs found via the RSE match well the analytic values given by Eq. (D2) even if the cut modes are not taken into account. In fact, in this case the relative error is still rather low, as can be seen in Fig. 13(b). Obviously, it is higher for the modes which are close to cut and does not improve for these modes with increasing $N$, the number of the RSs in the basis. When cut modes are included in the basis, the relative error scales as $1 / N^{3}$, almost uniformly for all the RSs, which is essentially the same as in the RSE used in the $k$ representation.

The total number of modes in the basis is given by $N_{\text {tot }}=$ $N+4 N_{c}=(1+F) N$, where we have introduced the factor

FIG. 17. (a) RS frequencies of a PC slab with $\epsilon=6, \alpha=0, \beta=$ $1, d=2 \pi a / 25$, and $p=0$, calculated for $F=1$ via the PC-RSE (red crosses). Unperturbed RSs and cut modes are also shown (black circles with dots and black dots, respectively). (b) Relative error of the PC-RSE compared to the RSE with $N \approx 4000$, taking the latter as "exact," calculated for different basis sizes as given.

$F$, the ratio of the number of cut modes to the number of RSs included. In Fig. 13(c) we show how the error depends on $F$ for a fixed $N_{\text {tot }}$. Higher values of $F$ imply more cut modes included in the basis at the expense of RSs. It is clear that larger values of $F$ give generally lower errors for the RSs close to the cut. However, modes with larger frequencies away from the cut are less accurately determined in this case, as the number of basis RSs $N$ reduces with $F$. We found that the value $F=1$ is close to the optimal one, as all RSs in a wide spectral range have a similar level of errors. We have made a similar study of the relative error in the case of the PC-RSE and found the same optimal value of $F$. Therefore, unless stated differently, the value of $F=1$ is used in all calculations throughout this paper.

\section{APPENDIX E: MODE CONTRIBUTIONS}

In addition to Fig. 7 of the main text, showing the expansion coefficients for a BIC-QGM pair, Figs. 14 and 15 show basis mode contributions for three other types of modes: FP, leaky, and cut modes. Similar to the BIC-QGM pair in Fig. 7, two perturbed FP RSs in Fig. 14 originate from a pair of 

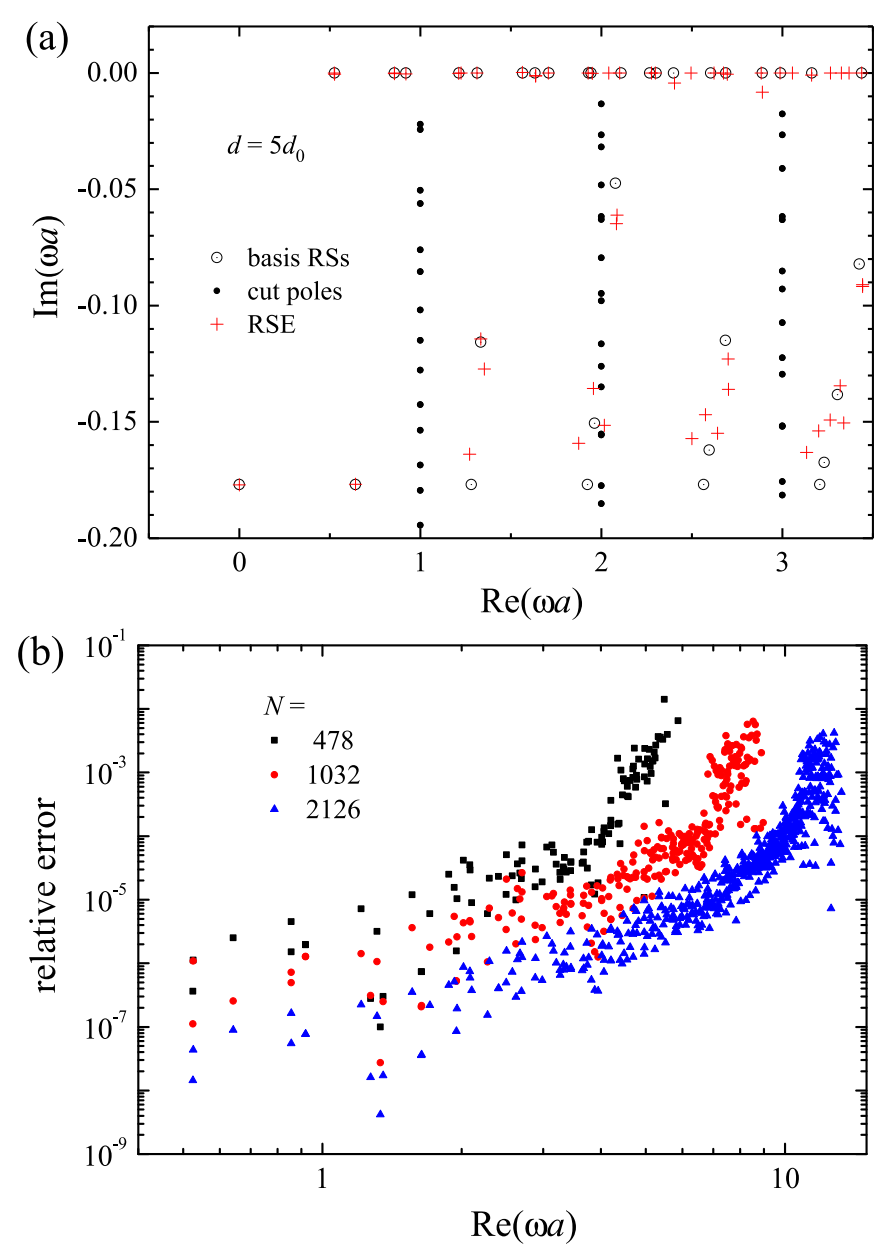

FIG. 18. As Fig. 17 but for $d=2 \pi a$.

degenerate unperturbed FP modes, and only one of them has a nonzero contribution of $m=0$ leaky modes. This makes the
$Q$ factor for that mode slightly lower than for the other one, since the mode is not affected by any $m=0$ basis states due to symmetry. Interestingly, both perturbed modes are almost equally strongly influenced by the pair of FP and a pair of WG basis modes matching the perturbed mode frequency.

Like in Fig. 7, the leaky and cut modes shown in Fig. 15 have a dominant contribution of only one basis mode (or one pair of modes), while the contribution of any other mode in the basis does not exceed a few per thousand.

\section{APPENDIX F: OTHER PARAMETERS}

We finally study the dependence of the RS frequencies calculated via the PC-RSE and their errors on the two structural parameters of the PC slab: the half-width of the core layer $b$ and the period of modulation $d$, while keeping $\epsilon=6, \alpha=0$, $\beta=1$, and $p=0$ as before.

Increasing $b$ from half-width $(b=a / 2)$ to the full-width value $(b=a)$ does not lead to any significant changes in the spectrum, as one can see in Fig. 16(a). The relative error is however getting sensitive to $b$ as $b \rightarrow a$. In fact, the error in Fig. 16(b) shows that the case of $b=0.95 a$ can produce up to an order of magnitude higher errors (relative to the SMM) compared to the system with $b=0.5 a$. The reason for this increase is related to the ML series changing its convergence properties on the borders of the system, which requires a further study. Presently, it prevents the PC-RSE from being used with exactly $b=a$.

Figures 17 and 18 show the RS frequencies and the relative error for the period of modulation $d$, respectively, five times smaller and five times larger than that used for Fig. $3\left(d_{0}=\right.$ $2 \pi a / 5)$. Such changes of the period change the spectrum of the RSs dramatically, so for instance, in the first case the number of RSs per cut is much larger than in Fig. 3 and in the second case, much smaller. Nevertheless, the PC-RSE is working equally well in all these cases, as we can see from almost the same level of errors.
[1] L. Liu, M. Pu, K. Yvind, and J. M. Hvam, Appl. Phys. Lett. 96, 051126 (2010).

[2] S. J. Mcnab, N. Moll, and Y. A. Vlasov, Opt. Express 11, 2927 (2003).

[3] A. R. McGurn, Phys. Rev. B 61, 13235 (2000).

[4] M. Bayindir, B. Temelkuran, and E. Ozbay, Appl. Phys. Lett. 77, 3902 (2000).

[5] E. Yablonovitch, T. J. Gmitter, and K. M. Leung, Phys. Rev. Lett. 67, 2295 (1991).

[6] D. M. Whittaker and I. S. Culshaw, Phys. Rev. B 60, 2610 (1999).

[7] S. G. Tikhodeev, A. L. Yablonskii, E. A. Muljarov, N. A. Gippius, and T. Ishihara, Phys. Rev. B 66, 045102 (2002).

[8] S. Fan and J. D. Joannopoulos, Phys. Rev. B 65, 235112 (2002).

[9] W. Zhou, D. Zhao, Y. Shuai, H. Yang, S. Chuwongin, A. Chadha, J. Seo, K. Wang, V. Liu, Z. Ma et al., Prog. Quantum Electron. 38, 1 (2014).

[10] G. Gamow, Z. Phys. 51, 204 (1928).

[11] A. F. J. Siegert, Phys. Rev. 56, 750 (1939).
[12] L. A. Weinstein, Open Resonators and Open Waveguides (Golden Press, Boulder, CO, 1969).

[13] R. W. Wood, Philos. Mag. 4, 396 (1902).

[14] M. B. Doost, W. Langbein, and E. A. Muljarov, Phys. Rev. A 87, 043827 (2013).

[15] S. V. Lobanov, G. Zoriniants, W. Langbein, and E. A. Muljarov, Phys. Rev. A 95, 053848 (2017).

[16] A. Gras, W. Yan, and P. Lalanne, Opt. Lett. 44, 3494 (2019).

[17] A. B. Akimov, N. A. Gippius, and S. G. Tikhodeev, JETP Lett. 93, 427 (2011).

[18] L. J. Armitage, M. B. Doost, W. Langbein, and E. A. Muljarov, Phys. Rev. A 89, 053832 (2014).

[19] A. O. Govorov, Z. Fan, P. Hernandez, J. M. Slocik, and R. R. Naik, Nano Lett. 10, 1374 (2010).

[20] T. Weiss, M. Mesch, M. Schäferling, H. Giessen, W. Langbein, and E. A. Muljarov, Phys. Rev. Lett. 116, 237401 (2016).

[21] T. Weiss, M. Schäferling, H. Giessen, N. A. Gippius, S. G. Tikhodeev, W. Langbein, and E. A. Muljarov, Phys. Rev. B 96, 045129 (2017).

[22] F. Vollmer and S. Arnold, Nat. Methods 5, 591 (2008). 
[23] F. Vollmer, D. Braun, A. Libchaber, M. Khoshsima, I. Teraoka, and S. Arnold, Appl. Phys. Lett. 80, 4057 (2002).

[24] M. Rosenblit, P. Horak, S. Helsby, and R. Folman, Phys. Rev. A 70, 053808 (2004).

[25] N. C. Frateschi and A. F. J. Levi, Appl. Phys. Lett. 66, 2932 (1995).

[26] P. Lalanne, W. Yan, K. Vynck, C. Sauvan, and J.-P. Hugonin, Laser Photon. Rev. 12, 1700113 (2018).

[27] E. A. Muljarov, W. Langbein, and R. Zimmermann, Europhys. Lett. 92, 50010 (2010).

[28] M. B. Doost, W. Langbein, and E. A. Muljarov, Phys. Rev. A 90, 013834 (2014).

[29] C. Sauvan, J.-P. Hugonin, I. S. Maksymov, and P. Lalanne, Phys. Rev. Lett. 110, 237401 (2013).

[30] D. Floess, M. Hentschel, T. Weiss, H.-U. Habermeier, J. Jiao, S. G. Tikhodeev, and H. Hiessen, Phys. Rev. X 7, 021048 (2017).

[31] M. B. Doost, W. Langbein, and E. A. Muljarov, Phys. Rev. A 85, 023835 (2012).

[32] L. J. Armitage, M. B. Doost, W. Langbein, and E. A. Muljarov, Phys. Rev. A 97, 049901(E) (2018).

[33] E. A. Muljarov and W. Langbein, Phys. Rev. B 93, 075417 (2016).

[34] E. A. Muljarov and T. Weiss, Opt. Lett. 43, 1978 (2018).

[35] N. W. Ashcroft and N. D. Mermin, Solid State Physics (Saunders College, Philadelphia, 1976), Chap. 8, pp. 132-140.

[36] L. C. Andreani and D. Gerace, Phys. Rev. B 73, 235114 (2006).

[37] M. Minkov and V. Savona, Sci. Rep. 4, 5124 (2014).

[38] W. Yan, R. Faggiani, and P. Lalanne, Phys. Rev. B 97, 205422 (2018).

[39] S. V. Lobanov, W. Langbein, and E. A. Muljarov, Phys. Rev. A 98, 033820 (2018).

[40] T. Weiss and E. A. Muljarov, Phys. Rev. B 98, 085433 (2018).

[41] Y. Akahane, T. Asano, B. S. Song, and S. Noda, Nature (London) 425, 944 (2003).
[42] P. Lalanne and E. Silberstein, Opt. Lett. 25, 1092 (2000).

[43] E. Silberstein, P. Lalanne, J.-P. Hugonin, and Q. Cao, J. Opt. Soc. Am. A 18, 2865 (2001).

[44] L. Li, J. Opt. A: Pure Appl. Opt. 5, 345 (2003).

[45] T. Weiss, N. A. Gippius, S. G. Tikhodeev, G. Granet, and H. Giessen, J. Opt. A: Pure Appl. Opt. 11, 114019 (2009).

[46] X. Gao, C. Hsu, B. Zhen, X. Lin, J. D. Joannopoulos, M. Soljaĉić, and H. Chen, Sci. Rep. 6, 31908 (2016).

[47] J. von Neumann and E. Wigner, Phys. Z. 30, 465 (1929).

[48] D. C. Marinica and A. G. Borisov, and S. V. Shabanov, Phys. Rev. Lett. 100, 183902 (2008).

[49] E. N. Bulgakov and A. F. Sadreev, Phys. Rev. B 78, 075105 (2008).

[50] N. Moiseyev, Phys. Rev. Lett. 102, 167404 (2009).

[51] Y. Plotnik, O. Peleg, F. Dreisow, M. Heinrich, S. Nolte, A. Szameit, and M. Segev, Phys. Rev. Lett. 107, 183901 (2011).

[52] G. B. Arfken and H. J. Weber, Mathematical Methods for Physicists, 5th ed. (Academic, San Diego, 2001), p. 448.

[53] D. A. Bykov and L. L. Doskolovich, J. Lightwave Technol. 31, 793 (2013).

[54] A. Taghizadeh and I. S. Chung, Appl. Phys. Lett. 111, 031114 (2017).

[55] E. N. Bulgakov and A. F. Sadreev, Phys. Rev. A 99, 033851 (2019).

[56] E. N. Bulgakov and A. F. Sadreev, Phys. Rev. A 90, 053801 (2014).

[57] B. Zhen, C. W. Hsu, L. Lu, A. D. Stone, and M. Soljaciić, Phys. Rev. Lett. 113, 257401 (2014).

[58] C. W. Hsu, B. Zhen, A. D. Stone, J. D. Joannopoulos, and M. Soljaĉić, Nat. Rev. Mater. 1, 16048 (2016).

[59] D. A. Bykov, E. A. Bezus, and L. L. Doskolovich, Phys. Rev. A 99, 063805 (2019).

[60] Z. F. Sadrieva, I. S. Sinev, K. L. Koshelev, A. Samusev, I. V. Iorsh, O. Takayama, R. Malureanu, A. A. Bogdanov, and A. V. Lavrinenko, ACS Photonics 4, 723 (2017). 